\title{
Synthesis, biological evaluation and molecular docking studies of some novel cyclopropane carbohydrazide derivatives as potential anticancer agents
}

\author{
PONNAPALLI VEERABHADRA SWAMY ${ }^{\mathrm{a}, \mathrm{b}, *}$, PULLAIAH CHINA KAMBHAMPATI ${ }^{\mathrm{a}}$, \\ KOTHAPALLI BONNOTH CHANDRASEKHAR ${ }^{\mathrm{c}}$, GUGULOTHU THIRUPATHI ${ }^{\mathrm{b}}$, \\ POMBALA SUJTHHA $^{\mathrm{d}}$, CHITYAL GANESH KUMAR ${ }^{\mathrm{d}, *}$ and \\ VEERAMACHANENI GANESH KUMAR ${ }^{\mathrm{e}}$ \\ ${ }^{a}$ Laxai Avanti Life Sciences, Lab\#9, ICICI Knowledge Park, Shameerpet, Turkapally Village, \\ Hyderabad, Telengana, 500 078, India \\ ${ }^{b}$ Department of Chemistry, Jawaharlal Nehru Technological University Hyderabad, \\ Hyderabad, Telangana, 500 085, India \\ 'Department of Chemistry, Jawaharlal Nehru Technological University Anantapuramu, \\ College of Engineering Anantapur, Andhra Pradesh, 500 085, India \\ ${ }^{\mathrm{d}}$ Medicinal Chemistry and Pharmacology Division, CSIR-Indian Institute of Chemical Technology, \\ Tarnaka, Hyderabad, 500 007, India

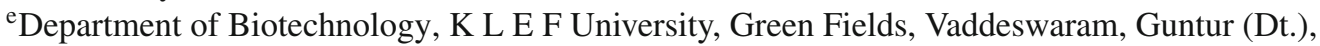 \\ Andhra Pradesh, 522 502, India \\ e-mail: ponnapalli74@yahoo.com; cgkumar5@gmail.com
}

MS received 6 November 2015; revised 19 March 2016; accepted 22 March 2016

\begin{abstract}
The synthesis of novel series of cyclopropane carbohydrazides is described via Knoevenagel condensation of 2-furfuraldehyde with malonic acid in five steps. Condensation of the key intermediate 2-(furan2-yl)cyclopropanecarbohydrazide (4) with heteroaryl/aryl aldehydes (a-t) in presence of $\mathrm{ZnO} \mathrm{NP}$ in ethanol resulted in substituted N- hetero/arylidene-2-(furan-2-yl) cyclopropane carbohydrazides (5a-t). These compounds were screened for their anticancer activity against a panel of four cancer cell lines and four compounds showed promising activity at micromolar concentration against all the tested cell lines with $\mathrm{IC}_{50}$ values ranging between 1.9-8.45 $\mu \mathrm{M}$. These compounds were further validated with in silico methods at the anticancer target, colchicine binding site.
\end{abstract}

Keywords. Anticancer activity; cyclopropane; carbohydrazides; 2-furfuraldehyde.

\section{Introduction}

Search for small molecules that connect to therapeutically imperative biological targets with high affinity and selectivity is the most important goal in modern bioorganic and medicinal chemistry. The reactivity of cyclopropanes permits them as adaptable intermediates in the synthesis of complex molecules, and these molecules are frequently engaged as versatile building blocks in organic syntheses. The cyclopropane ring is a significant structural part in many synthetic and natural compounds that demonstrate an extensive assortment of biological activities like antimicrobial, antiviral, antitumor, antiviral and herbicidal properties. ${ }^{1-14}$ Some derivatives of cyclopropane have shown potent

\footnotetext{
*For correspondence
}

anti-HIV activity as non-nucleoside reverse transcriptase inhibitors. ${ }^{15}$ Due to diversity of cyclopropane containing compounds with biological activity, chemists have tried to find novel and facile methods for synthesis of these compounds. ${ }^{16-22}$ This paper describes the synthesis, structural elucidation and molecular docking studies of a few new cyclopropane carbohydrazide analogues, prepared from commercially available 2-furfuraldehyde in five steps.

\section{Experimental}

\subsection{Materials and methods}

The dry solvents and the chemicals available commercially were used for the chemical process. Silica gel 60 F24 of Merck pre-coated plates were employed for their thin layer chromatography (TLC) analysis and 
the spots formed were visualized by UV-light. Merck silica gel (230-400) mesh was employed for flash column chromatography and the eluting solvents are mentioned in the procedures. Melting point (M.p.) was determined by Mel-temp apparatus. ${ }^{1} \mathrm{H}$ NMR spectra was recorded using Varian MR-400 MHz NMR devise. Chemical shifts are reported in $\delta$ parts per million (ppm) downfield from tetramethylsilane (TMS) as reference internal standard and the signals are reported as s (singlet), d (doublet), dd (doublet of doublet), t (triplet), q (quartet), $\mathrm{m}$ (multiple) and coupling constants in $\mathrm{Hz}$. The data related to mass spectra was recorded on Agilent ion trap MS. Infrared (IR) spectra were recorded on a Perkin Elmer FT-IR spectrometer.

\subsection{Synthesis of intermediate compounds and final compounds}

2.2a Synthesis of (E)-3-(furan-2-yl)acrylic acid (1): To a solution of malonic acid $(33.67 \mathrm{mg}, 0.15 \mathrm{mmol})$ in pyridine $(3 \mathrm{~mL})$ were added piperidine $(44.2 \mathrm{mg}, 0.52$ $\mathrm{mmol}$ ) and furan-2-carbaldehyde $(500 \mathrm{mg}, 5.20 \mathrm{mmol})$ and stirred at $110^{\circ} \mathrm{C}$ for $2 \mathrm{~h}$. TLC showed completion of reaction (TLC system: 20\% EtOAc in Hexane, Rf: 0.5$)$. Then the reaction mixture was concentrated in vacuo, water $(10 \mathrm{~mL})$ was added and neutralized with $6 \mathrm{~N} \mathrm{HCl}$. The precipitate obtained was filtered, washed with water $(3 \mathrm{~mL})$, dried under vacuum to obtain 3(furan-2-yl)acrylic acid (1). Brown solid; Yield: 100 mg, 55\%; M.p. $78-82^{\circ} \mathrm{C}$; IR (KBr): $v_{\max } / \mathrm{cm}^{-1} 3327$ (-COOH stretching); ${ }^{1} \mathrm{H}$ NMR (400 MHz, DMSO- $\left.d_{6}\right) \delta$ 12.3 (s, 1H, -OH), 7.81 (s, 1H, Ar-H), 7.37 (d, $J=15.8$ $\mathrm{Hz}, 1 \mathrm{H},-\mathrm{C}=\mathrm{CH}-), 6.91(\mathrm{~d}, J=3.2 \mathrm{~Hz}, 1 \mathrm{H}, \mathrm{Ar}-\mathrm{H}), 6.61$ $(\mathrm{dd}, J=1.4,3.6 \mathrm{~Hz}, 1 \mathrm{H}, \mathrm{Ar}-\mathrm{H}), 6.15(\mathrm{~d}, J=15.8 \mathrm{~Hz}$, $1 \mathrm{H},-\mathrm{C}=\mathrm{CH}-) ;{ }^{13} \mathrm{C}$ NMR $\left(100 \mathrm{MHz}\right.$ DMSO- $\left.d_{6}\right) \delta 167.3$ $(\mathrm{C}=\mathrm{O}), 150.3,145.6(\mathrm{Ar}-\mathrm{C}), 130.8,116.1(-\mathrm{HC}=\mathrm{CH}-)$, 115.3, 112.6 (Ar-C); APCI-MS $m / z: 137[\mathrm{M}-\mathrm{H}]^{+}$.

2.2b Synthesis of ethyl 3-(furan-2-yl)acrylate (2): To a solution of (E)-3 -(furan-2-yl)acrylic acid (1) (500 $\mathrm{mg}, 3.60 \mathrm{mmol})$, in ethanol $(5 \mathrm{~mL})$ was added conc. $\mathrm{H}_{2} \mathrm{SO}_{4}$ (catalytic). The mixture was heated to reflux and maintained for $16 \mathrm{~h}$. TLC showed completion of starting material (TLC system: 10\% EtOAc in Hexane, Rf: $0.5)$. The reaction mixture was cooled to room temperature and concentrated to obtain a residue. The residue was diluted with EtOAc $(20 \mathrm{~mL})$, washed with $\mathrm{NaHCO}_{3}$ solution $(10 \mathrm{~mL})$, water $(10 \mathrm{~mL})$, dried over anhydrous sodium sulphate, and concentrated to obtain the crude product. The crude product was purified by silica gel column chromatography to obtain ethyl 3-(furan-2yl)acrylate (2). Colorless liquid; Yield: $100 \mathrm{mg}$, 49\%; IR (neat): $v_{\max } / \mathrm{cm}^{-1} 1709\left(\mathrm{C}=\mathrm{O}\right.$ stretching); ${ }^{1} \mathrm{H}$ NMR
(400 MHz, DMSO- $\left.d_{6}\right) \delta 7.82(\mathrm{~s}, 1 \mathrm{H}, \mathrm{Ar}-\mathrm{H}), 7.44(\mathrm{~d}$, $J=15.8 \mathrm{~Hz}, 1 \mathrm{H},-\mathrm{C}=\mathrm{CH}-), 6.95(\mathrm{~d}, J=3.3 \mathrm{~Hz}, 1 \mathrm{H}$, Ar-H), 6.61 (dd, $J=1.7,3.2 \mathrm{~Hz}, 1 \mathrm{H}, \mathrm{Ar}-\mathrm{H}), 6.25$ (d, $J=15.8 \mathrm{~Hz}, 1 \mathrm{H},-\mathrm{C}=\mathrm{CH}-), 4.15(\mathrm{q}, J=7.0 \mathrm{~Hz}, 2 \mathrm{H}$, $\left.-\mathrm{OCH}_{2}\right), 1.23\left(\mathrm{t}, J=7.0 \mathrm{~Hz}, 3 \mathrm{H},-\mathrm{CH}_{3}\right) ;{ }^{13} \mathrm{C} \mathrm{NMR}(100$ $\left.\mathrm{MHz}, \mathrm{DMSO}-d_{6}\right) \delta 165.9(\mathrm{C}=\mathrm{O}), 150.1,145.8(\mathrm{Ar}-\mathrm{C})$, $115.8(-\mathrm{HC}=\mathrm{CH}-), 114.7,112.6(\mathrm{Ar}-\mathrm{C}), 59.9\left(-\mathrm{CH}_{2}\right)$, $14\left(-\mathrm{CH}_{3}\right)$; GC-MS $m / z: 166.1\left(\mathrm{M}^{+}\right)$.

2.2c Synthesis of ethyl 2-(furan-2-yl)cyclopropanecarboxylate (3): To a suspension of $\mathrm{NaH}(722 \mathrm{mg}$, $0.03 \mathrm{~mol})$, in dry DMSO $(3 \mathrm{~mL})$, at $0^{\circ} \mathrm{C}$, trimethyl sulfoxonium iodide $(9.93 \mathrm{~g}, 0.03 \mathrm{~mol})$ was added and stirred for $1 \mathrm{~h}$. To this (E)-ethyl 3-(furan-2-yl)acrylate (2) $(5 \mathrm{~g}, 0.03 \mathrm{~mol})$ was added and stirred at $\mathrm{rt}$ for $1 \mathrm{~h}$. TLC showed the reaction was completed (TLC system: 10\% EtOAc in Hexane, Rf: 0.5). Reaction mass was diluted with ice water $(30 \mathrm{~mL})$, extracted into EtOAc $(2 \times 15 \mathrm{~mL})$, dried over $\mathrm{Na}_{2} \mathrm{SO}_{4}$, concentrated under reduced pressure and purified by silica gel column (3\% EtOAc in Hexane as eluent) to obtain ethyl 2-(furan2-yl)cyclopropanecarboxylate (3). Pale yellow liquid; Yield: $500 \mathrm{mg}$, 46\%; IR (neat): $v_{\max } / \mathrm{cm}^{-1} 1727$ (-C=Ostretching); ${ }^{1} \mathrm{H}$ NMR (400 MHz, DMSO- $\left.d_{6}\right) \delta 7.24$ (dd, $J=0.7,1.7 \mathrm{~Hz}, 1 \mathrm{H}$, Ar-H), $6.26(\mathrm{dd}, J=1.8, J=3.2$ $\mathrm{Hz}, 1 \mathrm{H}, \mathrm{Ar}-\mathrm{H}), 6.05(\mathrm{~d}, J=3.2 \mathrm{~Hz}, 1 \mathrm{H}, \mathrm{Ar}-\mathrm{H}), 4.18$ (q, $\left.J=1.7 \mathrm{~Hz}, 2 \mathrm{H},-\mathrm{OCH}_{2}-\right), 2.50(\mathrm{~m}, 1 \mathrm{H}$, cyc-CH-), 1.99 (m, 1H, cyc-CH-), 1.51 (m, 1H, cyc-CH-), 1.49 (m, 1H, cyc-CH-), 1.29 (t, $\left.J=3.8 \mathrm{~Hz}, 3 \mathrm{H},-\mathrm{CH}_{3}\right)$; ${ }^{13} \mathrm{C}$ NMR (100 MHz, DMSO- $\left.d_{6}\right) \delta 172.7(\mathrm{C}=\mathrm{O})$, 153.3, 140.9, 110.3, 105.1 (Ar-C), $60.6\left(\mathrm{CH}_{2}\right), 21.9$, 19.2 (Cyclopropane), $14.6\left(\mathrm{CH}_{3}\right), 14.2$ (Cyclopropane); ESI-MS, $m / z: 108.98[\mathrm{M}+\mathrm{H}]^{+}$.

2.2d Synthesis of 2-(furan-2-yl)cyclopropanecarbohydrazide (4): To a stirred solution of ethyl 2-(furan2-yl)cyclopropanecarboxylate $(1 \mathrm{~g}, 5.50 \mathrm{mmol})$ in 1,4dioxane $(10 \mathrm{~mL})$, hydrazine hydrate $(2.1 \mathrm{~mL}, 66.60$ mmol) was added and stirred at $100^{\circ} \mathrm{C}$ for $24 \mathrm{~h}$. After completion of starting material (TLC system: $5 \% \mathrm{MeOH}$ in DCM, Rf: 0.3) the reaction mixture was minimized to $10 \%$ volume by evaporation under reduced pressure, and then cooled. The solid obtained was filtered and dried to get 4-(furan-2-yl)-1H-pyrrole3-carbohydrazide (4). Off white solid; Yield: $650 \mathrm{mg}$, 70\%; M.p. 118-124 ${ }^{\circ}$; IR (KBr): $v_{\max } / \mathrm{cm}^{-1} 3274(\mathrm{NH}$ stretching), 3191, $3152\left(\mathrm{NH}_{2}\right.$ stretching $), 1632(\mathrm{C}=\mathrm{O}$ stretching); ${ }^{1} \mathrm{H}$ NMR (400 MHz, DMSO- $\left.d_{6}\right) \delta 9.20$ (brs, $1 \mathrm{H},-\mathrm{NH}-), 7.46$ (dd, $J=0.7,1.7 \mathrm{~Hz}, 1 \mathrm{H}, \mathrm{Ar}-\mathrm{H}), 6.34$ (dd, $J=1.9,3.2 \mathrm{~Hz}, 1 \mathrm{H}$, Ar-H), $6.14(\mathrm{~d}, J=3.1 \mathrm{~Hz}$, $1 \mathrm{H}, \mathrm{Ar}-\mathrm{H}), 4.21$ (bs, 2H, $-\mathrm{NH}_{2}$ ), 2.22 (m, 1H, cyc-CH-), $1.81(\mathrm{~m}, 1 \mathrm{H}$, cyc-CH-), $1.22(\mathrm{~m}, 1 \mathrm{H}$, cyc-CH-), $1.16(\mathrm{~m}$, $1 \mathrm{H}$, cyc-CH-); ${ }^{13} \mathrm{C}$ NMR $\left(100 \mathrm{MHz}, \mathrm{DMSO}-d_{6}\right) \delta 170.1$ 
$(\mathrm{C}=\mathrm{O}), 153.9,141.2,110.5,104.8$ (Ar-C), 21.5, 16.7, 12.3 (Cyclopropane); ESI-MS, $m / z: 166.97[\mathrm{M}+\mathrm{H}]^{+}$.

2.2e General procedure for the preparation of 5a-t using conc. $\mathrm{HCl}$ : A mixture of $\mathbf{4}(10 \mathrm{mmol})$, appropriate aldehyde $(10 \mathrm{mmol})$ from the list a-t, conc. $\mathrm{HCl}$ $(1 \mathrm{mmol})$ and ethanol $(25 \mathrm{~mL})$ was stirred at $85^{\circ} \mathrm{C}$ for 5-6 h. After completion of reaction (monitored by TLC), the reaction mixture was cooled to rt. The obtained precipitate was filtered, washed with n-pentane and dried to obtain crude compounds 5a-t. The obtained crude compounds were recrystallized from ethanol to afford pure 5a-t.

2.2f General procedure for the preparation of 5a-t using PEG-400: A mixture of appropriate aldehyde $(10 \mathrm{mmol})$ from the list a-t, compound $4(10 \mathrm{mmol})$, PEG-400 (25 mL) was stirred at rt for 7-8 h. After completion of reaction (monitored by TLC), the mixture was poured into ice cold water $(50 \mathrm{~mL})$ and neutralized with sodium bicarbonate solution. The separated solid was filtered, washed with water $(100 \mathrm{~mL})$ and dried to obtain crude products 5a-t. The obtained crude compounds were recrystallized from ethanol to afford pure 5a-t.

$2.2 \mathrm{~g}$ General procedure for the preparation of 5(a-t) using PEG-600: A mixture of appropriate aldehyde $(10 \mathrm{mmol})$ from the list $\mathbf{a}-\mathbf{t}$, compound 4 (10 $\mathrm{mmol})$, PEG-600 (25 mL) was stirred at room temperature for 4-5 h. After completion of reaction (monitored by TLC), the mixture was poured into ice cold water $(50 \mathrm{~mL})$ and neutralized with sodium bicarbonate solution. The separated solid was filtered, washed with water $(100 \mathrm{~mL})$ and dried to obtain crude products 5a-t. The obtained crude compounds were recrystallized from ethanol to afford pure 5a-t.

\section{2h General procedure for the preparation of $\mathbf{5 ( a - t )}$} using L-tyrosine: A mixture of appropriate aldehyde (10 mmol) from the list a-t, compound 4 (10 mmol), L-tyrosine $(2 \mathrm{mmol})$ and water $(25 \mathrm{~mL})$ was stirred at room temperature for 2-3 h. After completion of reaction (monitored by TLC), the mixture was poured into ice cold water $(50 \mathrm{~mL})$ and separated solid was filtered, washed with water $(100 \mathrm{~mL})$ and dried to obtain crude products 5a-t which were purified by recrystallization in ethanol to afford pure compounds.

$2.2 \mathrm{i}$ General procedure for the preparation of $\mathbf{5}(\boldsymbol{a}-\boldsymbol{t})$ using microwave irradiation: A mixture of appropriate aldehyde $(10 \mathrm{mmol})$ from the list $\mathbf{a}-\mathbf{t}$, compound $\mathbf{4}$
$(10 \mathrm{mmol})$ and water $(10 \mathrm{~mL})$ in a $10 \mathrm{~mL}$ microwave vial was introduced into the microwave oven and irradiated for $30 \mathrm{~min}$ to $1 \mathrm{~h}$. After completion of reaction (monitored by TLC), the reaction mixture was cooled to rt, obtained precipitate was filtered, washed with npentane and dried to obtain crude product 5a-t which was purified by recrystallization in ethanol to afford pure compounds.

$2.2 \mathrm{j}$ General procedure for the preparation of 5(a-t) using ZnO NPs: A mixture of appropriate aldehyde $(10 \mathrm{mmol})$ from the list a-t, compound 4 (10 $\mathrm{mmol}), \mathrm{ZnO}$ Nano particles $(2 \mathrm{mmol})$ and ethanol (25 $\mathrm{mL}$ ) was stirred at $\mathrm{rt}$ for $30 \mathrm{~min}$ to $1 \mathrm{~h}$. After completion of reaction (monitored by TLC), the reaction mixture was filtered through a nanofiltration membrane (Make: Synder, Model: NFS 100-250Da). The filtrate was evaporated under reduced pressure and dried to obtain crude product 5a-t which was further purified by recrystallization in ethanol to afford pure compounds.

(E) - $N^{\prime}$ - (4-fluorobenzylidene)-2-(furan-2-yl)cyclopropanecarbohydrazide (5a): Off-white solid; Yield: $82 \mathrm{mg}, 83 \%$; M.p. $152-158^{\circ} \mathrm{C}$; IR (KBr): $v_{\max } / \mathrm{cm}^{-1}$ 3180 (NH stretching), $1664(\mathrm{C}=\mathrm{O}$ stretching), 1606 $\left(\mathrm{C}=\mathrm{N}\right.$ stretching); ${ }^{1} \mathrm{H}$ NMR (400 MHz, DMSO- $\left.d_{6}\right) \delta$ $11.68(* 11.51, \mathrm{~s}, 1 \mathrm{H},-\mathrm{CO}-\mathrm{NH}-\mathrm{N}-), 8.15(* 8.03, \mathrm{~s}$, $1 \mathrm{H},-\mathrm{N}=\mathrm{CH}-), 7.72(\mathrm{~m}, 2 \mathrm{H}, \mathrm{Ar}-\mathrm{H}), 7.49(\mathrm{~d}, J=3.2$ $\mathrm{Hz}, 1 \mathrm{H}, \mathrm{Ar}-\mathrm{H}), 7.24$ (q, $J=8.7 \mathrm{~Hz}, 2 \mathrm{H}, \mathrm{Ar}-\mathrm{H}), 6.36$ $(\mathrm{t}, J=1.3 \mathrm{~Hz}, 1 \mathrm{H}, \mathrm{Ar}-\mathrm{H}), 6.22(\mathrm{dd}, J=3.1,6.6 \mathrm{~Hz}$, $1 \mathrm{H}, \mathrm{Ar}-\mathrm{H}), 2.90-2.88$ (* 2.39-2.35, m, $1 \mathrm{H}$, cyc-CH-Ar), 2.48-2.43 (*2.0-1.96, m, 1H, cyc-CH-CO-), 1.45-1.31 $\left(\mathrm{m}, 1 \mathrm{H},-\mathrm{CH}_{2}-\right) ;{ }^{13} \mathrm{C}$ NMR $\left(100 \mathrm{MHz}, \mathrm{DMSO}-d_{6}\right) \delta$ $172.2(\mathrm{C}=\mathrm{O}), 167.1($ Ar-C $), 153.7(* 153.6)(\mathrm{C}=\mathrm{N})$, 144.8, 141.4, 130.8 (* 130.6), 129.1 (* 129), 128.8 (* 128.7), 115.9 (* 115.8), 110.6, 105.1 (Ar-C), 22.4 (* 19.3), 18.3 (* 17.8), 14.1 (* 13) (Cyclopropane); ESI-MS, $m / z: 271.1[\mathrm{M}-\mathrm{H}]^{+}$.

(E)- $N^{\prime}$-(3,4-difluorobenzylidene)-2-(furan-2-yl)cyclopropanecarbohydrazide $(\mathbf{5 b})$ : Off-white solid; Yield: $79 \mathrm{mg}$; 64\%; M.p. $148-152^{\circ} \mathrm{C}$; IR (KBr): $v_{\max } / \mathrm{cm}^{-1}$ 3179 (NH stretching), 1666 ( $\mathrm{C}=\mathrm{O}$ stretching), 1614 $\left(\mathrm{C}=\mathrm{N}\right.$ stretching); ${ }^{1} \mathrm{H}$ NMR (400 MHz, DMSO- $\left.d_{6}\right) \delta$ 11.79 (* 11.62, s, 1H, -CO-NH-N-), 8.13 (* 8.00, s, $1 \mathrm{H},-\mathrm{N}=\mathrm{CH}-), 7.70(\mathrm{dd}, J=2.0,3.5 \mathrm{~Hz}, 1 \mathrm{H}, \mathrm{Ar}-\mathrm{H})$, 7.58-7.43 (m, 3H, Ar-H), 6.37 (d, $J=1.5 \mathrm{~Hz}, 1 \mathrm{H}$, Ar-H), 6.23 (dd, $J=4.1,7.8 \mathrm{~Hz}, 1 \mathrm{H}, \mathrm{Ar}-\mathrm{H}), 2.94-2.9$ (*2.39-2.35, m, cyc-CH-Ar), 2.48-2.40 (* 2.01-1.96, m, $1 \mathrm{H}$, cyc-CH-CO-), $1.44-1.31$ (m, $2 \mathrm{H}$, cyc- $\left.\mathrm{CH}_{2-}\right) ;{ }^{13} \mathrm{C}$ NMR (100 MHz, DMSO- $\left.d_{6}\right) \delta 172.4(\mathrm{C}=\mathrm{O}), 167.2$ (Ar-C), $153.6(* 153.5)(\mathrm{C}=\mathrm{N}), 143.7,141.3,132.1$, $124.1(* 123.9), 118$ (* 117.8), $115.3(* 115.1), 115.1$ 
(* 114.9), 110.5, 105.1 (Ar-C), 22.3 (* 19.2), 18.4 (* 17.8), $14.1(* 13.0)$ (Cyclopropane); ESI-MS, $m / z$ : $291.25[\mathrm{M}+\mathrm{H}]^{+}$.

(E)- $N^{\prime}$-(3,5-difluorobenzylidene)-2-(furan-2-yl)cyclopropanecarbohydrazide (5c): Off-white solid; Yield: 84 mg; 68\%; M.p. $162-168^{\circ} \mathrm{C}$; IR (KBr): $v_{\max } / \mathrm{cm}^{-1} 3181$ $(\mathrm{NH}$ stretching $), 1663(\mathrm{C}=\mathrm{O}$ stretching $), 1605(\mathrm{C}=\mathrm{N}$ stretching); ${ }^{1} \mathrm{H}$ NMR (400 MHz, DMSO- $\left.d_{6}\right) \delta 11.88$ (* $11.71, \mathrm{~s}, 1 \mathrm{H},-\mathrm{CO}-\mathrm{NH}-\mathrm{N}-), 8.15(* 8.01, \mathrm{~s}, 1 \mathrm{H}$, $-\mathrm{N}=\mathrm{CH}-), 7.50(\mathrm{~s}, 1 \mathrm{H}$, Ar-H) $7.39-7.31(\mathrm{~m}, 2 \mathrm{H}$, Ar-H), 7.28-7.23 (m, 1H, Ar-H), 6.37 (t, $J=2.2 \mathrm{~Hz}$, $1 \mathrm{H}$, Ar-H), $6.23(\mathrm{dd}, J=3.1,7.6 \mathrm{~Hz}, 1 \mathrm{H}, \mathrm{Ar}-\mathrm{H})$, 2.92-2.90 (* 2.39-2.36, m, 1H, cyc-CH-Ar), 2.49-2.40 (* 2.02-1.98, m, 1H, cyc-CH-CO-), 1.45-1.34 (m, 2H, cyc- $\left.\mathrm{CH}_{2-}\right) ;{ }^{13} \mathrm{C}$ NMR (100 MHz, DMSO- $\left.d_{6}\right) \delta 172.5$ $(\mathrm{C}=\mathrm{O}), 167.3,163.8(* 163.7), 161.3(* 161.1)(\mathrm{Ar}-\mathrm{C})$, $153.6(* 153.4)(\mathrm{C}=\mathrm{N}), 143.4,141.5(* 141.3), 140.8$, 110.5, 109.8 (* 109.6), 105.2, 104.7 (Ar-C), 22.4 (* 19.3), 18.5 (* 18), 14.2 (* 13.1) (Cyclopropane); ESI-MS, $m / z: 289.1[\mathrm{M}-\mathrm{H}]^{+}$.

(E)-N' -(4-fluoro-3-nitrobenzylidene)-2 -(furan-2-yl) cyclopropanecarbohydrazide (5d): Light yellow solid; M.p. $196-200^{\circ} \mathrm{C}$; Yield: $95 \mathrm{mg}, 71 \%$; IR (KBr): $v_{\max } / \mathrm{cm}^{-1} 3186$ ( $\mathrm{NH}$ stretching), $1662(\mathrm{C}=\mathrm{O}$ stretching), $1615\left(\mathrm{C}=\mathrm{N}\right.$ stretching); ${ }^{1} \mathrm{H}$ NMR $(400 \mathrm{MHz}$, DMSO$\left.d_{6}\right) \delta 11.91(* 11.73, \mathrm{~s}, 1 \mathrm{H},-\mathrm{CO}-\mathrm{NH}-\mathrm{N}-), 8.44-8.42$ (* 8.37-8.34, dd, $J=2.1,7.4 \mathrm{~Hz}, 1 \mathrm{H}$, Ar-H), 8.23 (* 8.12, s, $1 \mathrm{H},-\mathrm{N}=\mathrm{CH}-), 8.09-8.05(\mathrm{~m}, 1 \mathrm{H}, \mathrm{Ar}-\mathrm{H})$, 7.67-7.59 (m, 1H, Ar-H), 7.50 (s, 1H, Ar-H), 6.37 (dd, $J=1.9,3.0 \mathrm{~Hz}, 1 \mathrm{H}, \mathrm{Ar}-\mathrm{H}), 6.25-6.22(\mathrm{~m}, 1 \mathrm{H}, \mathrm{Ar}-\mathrm{H})$, 2.94-2.89 (* 2.39-2.36, m, 1H, cyc-CH-Ar), 2.49-2.40 (* 2.04-2.00, m, 1H, cyc-CH-CO-), 1.5-1.3 (m, 2H, cyc- $\left.\mathrm{CH}_{2-}\right) ;{ }^{13} \mathrm{C}$ NMR (100 MHz, DMSO- $\left.d_{6}\right) \delta 172.4$ $(\mathrm{C}=\mathrm{O}), 167.4(\mathrm{Ar}-\mathrm{C}), 153.6(* 153.4)(\mathrm{C}=\mathrm{N}), 142.6$, 141.5 (* 141.3), 134.1 (* 134), 133.6 (* 133.5), 131.8 (* 131.5), $124(* 123.8), 119.1(* 118.9), 110.6,105.22$ (* 105.26) (Ar-C), 22.4 (* 19.2), 18.5 (* 18), 14.1 (* 13.1) (Cyclopropane); ESI-MS, $m / z: 316.9$ [M-H] ${ }^{+}$.

(E)-N'-(4-fluoro-2-methoxybenzylidene)-2-(furan-2-yl) cyclopropanecarbohydrazide (5e): Off-white solid; Yield: $55 \mathrm{mg}, 61 \%$; M.p. $170-172^{\circ} \mathrm{C}$; IR $(\mathrm{KBr})$ : $v_{\max } / \mathrm{cm}^{-1} 3210(\mathrm{NH}$ stretching $), 1656(\mathrm{C}=\mathrm{O}$ stretching), $1608\left(\mathrm{C}=\mathrm{N}\right.$ stretching); ${ }^{1} \mathrm{H}$ NMR $(400 \mathrm{MHz}$, DMSO- $\left.d_{6}\right) \delta 11.66$ (*11.46, s, $\left.1 \mathrm{H},-\mathrm{CO}-\mathrm{NH}-\mathrm{N}-\right), 8.41$ (* 8.29, s, $1 \mathrm{H},-\mathrm{N}=\mathrm{CH}-), 7.80-7.72(\mathrm{~m}, 1 \mathrm{H}, \mathrm{Ar}-\mathrm{H})$, $7.50(\mathrm{~d}, J=0.6 \mathrm{~Hz}, 1 \mathrm{H}, \operatorname{Ar}-\mathrm{H}), 7.02-6.98(\mathrm{~m}, 1 \mathrm{H}$, Ar-H), 6.86-6.78 (m, 1H, Ar-H), 6.37-6.36 (d, $J=1.8$, $3.2 \mathrm{~Hz}, 1 \mathrm{H}, \mathrm{Ar}-\mathrm{H}), 6.23-6.21(\mathrm{dd}, J=3.2,6.2 \mathrm{~Hz}, 1 \mathrm{H}$, Ar-H), $3.85\left(* 3.83, \mathrm{~s},-\mathrm{OCH}_{3}\right), 2.89-2.88(* 2.48-2.45$, $\mathrm{m}$, cyc-CH-ArH), 2.49-2.48 (* 1.93-1.91, m, $1 \mathrm{H}$, cyc-CH-CO), $1.42-1.30$ (m, $2 \mathrm{H}$, cyc- $\left.\mathrm{CH}_{2-}\right) ;{ }^{13} \mathrm{C} \mathrm{NMR}$ $\left(100 \mathrm{MHz}, \mathrm{DMSO}-d_{6}\right) \delta 172.1(\mathrm{C}=\mathrm{O}), 166.8,158.9$ $(* 158.8)($ Ar-C), 153.7 $(* 153.5)(\mathrm{C}=\mathrm{N}), 141.4(* 141.3)$, 138.2, $126.9(* 126.7), 118.7,110.5,107.7(* 107.4)$, $105.1,100.1(* 99.8)(\mathrm{Ar}-\mathrm{C}), 56.18(* 56.14)\left(-\mathrm{OCH}_{3}\right)$, $22.3(* 19.3), 18.3(* 17.6), 14(* 12.9)$ (Cyclopropane); ESI-MS, $m / z: 302.9[\mathrm{M}+\mathrm{H}]^{+}$.

(E)-2-(furan-2-yl)- $N^{\prime}$-(4-(trifluoromethyl)benzylidene) cyclopropanecarbohydrazide (5f): Off-white solid; Yield: $70 \mathrm{mg}, 72 \%$; M.p. $108-112^{\circ} \mathrm{C}$; IR (KBr): $v_{\max } / \mathrm{cm}^{-1} 3202$ ( $\mathrm{NH}$ stretching), $1663(\mathrm{C}=\mathrm{O}$ stretching), $1551\left(\mathrm{~N}=\mathrm{C}\right.$ stretching); ${ }^{1} \mathrm{H}$ NMR $(400 \mathrm{MHz}$, DMSO$\left.d_{6}\right) \delta 11.87(* 11.70$, brs, $1 \mathrm{H},-\mathrm{CO}-\mathrm{NH}-\mathrm{N}-), 8.22$ (*8.11, s, $1 \mathrm{H},-\mathrm{N}=\mathrm{CH}-), 7.90-7.88(\mathrm{~d}, J=8.1 \mathrm{~Hz}, 1 \mathrm{H}$, Ar-H), 7.85-7.83 (d, $J=8.2, \mathrm{~Hz}, 1 \mathrm{H}, \mathrm{Ar}-\mathrm{H}), 7.79-7.54$ (t, $J=8.8 \mathrm{~Hz}, 2 \mathrm{H}, \mathrm{Ar}-\mathrm{H}), 7.50(\mathrm{~d}, J=3.3 \mathrm{~Hz}, 1 \mathrm{H}$, Ar-H), 6.38-6.37 * 6.34-6.22, d, $J=1.5, \mathrm{~Hz}, 1 \mathrm{H}$, Ar-H), 6.25-6.23 (* 6.15-6.14, dd, $J=3.1,7.2 \mathrm{~Hz}$, $1 \mathrm{H}, \mathrm{Ar}-\mathrm{H}), 2.94-2.89$ (* 2.24-2.21, m, cyc-CH-CO-), 2.41-2.38 (* 2.03-1.99, m, cyc-CH-CO-), 1.46-1.32 (m, 2H, cyc- $\left.\mathrm{CH}_{2}-\right) ;{ }^{13} \mathrm{C}$ NMR (100 MHz, DMSO- $d_{6}$ ) $\delta 172.4(\mathrm{C}=\mathrm{O}), 170.1,167.3(\mathrm{Ar}-\mathrm{C}), 153.6(* 153.4)$ $(\mathrm{C}=\mathrm{N}), 144.2,141.8(* 141.5), 141.3(* 141.1), 138.2$ (*138), $127.5(* 127.2), 125.6(* 125.3), 110.5,105.24$ (* 105.20) (Ar-C), $104.7\left(\mathrm{CF}_{3}\right), 22.4$ (* 21.5), 18.5 (* 17.9), 14.1 (* 12.2) (Cyclopropane); ESI-MS, $m / z$ : $320.9[\mathrm{M}-\mathrm{H}]^{+}$.

(E)-2-(furan-2-yl)-N'-(4-hydroxybenzylidene)cyclopropanecarbohydrazide (5g): Yellow solid; Yield: 68 mg, 60\%; M.p. $76-82^{\circ} \mathrm{C}$; IR (KBr): $v_{\max } / \mathrm{cm}^{-1} 3373$ (OH stretching), $3215(\mathrm{NH}$ stretching), $1654(\mathrm{C}=\mathrm{O}$ stretching), $1604\left(\mathrm{C}=\mathrm{N}\right.$ stretching); ${ }^{1} \mathrm{H}$ NMR (400 $\left.\mathrm{MHz}, \mathrm{DMSO}-d_{6}\right) \delta 11.46(* 11.29, \mathrm{~s}, 1 \mathrm{H},-\mathrm{CO}-\mathrm{NH}-$ $\mathrm{N}-), 8.53(* 7.50, \mathrm{~s}, 1 \mathrm{H}, \mathrm{Ar}-\mathrm{OH}), 8.03(* 7.93, \mathrm{~s}, 1 \mathrm{H}$, $-\mathrm{N}=\mathrm{CH}-), 7.68-7.66(\mathrm{~d}, J=8.6 \mathrm{~Hz}, 1 \mathrm{H}, \mathrm{Ar}-\mathrm{H}), 7.50-$ 7.48 (m, 1H, Ar-H), 7.44 (d, $J=8.5 \mathrm{~Hz}, 1 \mathrm{H}, \mathrm{Ar}-\mathrm{H})$, $6.82(\mathrm{~d}, J=8.5 \mathrm{~Hz}, 1 \mathrm{H}, \mathrm{Ar}-\mathrm{H}), 6.85-6.81$ (d, $J=10.5$ $\mathrm{Hz}, 1 \mathrm{H}, \mathrm{Ar}-\mathrm{H}), 6.22$ (m, 2H, Ar-H), 2.90-2.87 (* 2.362.32, m, cyc-CH-Ar), 2.46-2.42 (* 1.97-1.94, m, 1H, cyc-CH-CO-), 1.41-1.25 (m, 2H, cyc- $\left.\mathrm{CH}_{2-}\right) ;{ }^{13} \mathrm{C}$ NMR $\left(100 \mathrm{MHz}, \mathrm{DMSO}-d_{6}\right) \delta 171.9(\mathrm{C}=\mathrm{O}), 166.7,160.2$ $($ Ar-C), $153.8(* 153.7)(\mathrm{C}=\mathrm{N}), 146.3,141.4(* 141.3)$, 130.0, 128.7 (* 128.3), $125.1(* 125), 115.7$ (* 115.6), 110.6, 105.1 (Ar-C), 22.3 (* 19.3), 18.2 (* 17.6), 14 (*12.8) (Cyclopropane); ESI-MS, $m / z: 271.21[\mathrm{M}+\mathrm{H}]^{+}$.

(E)-2-(furan-2-yl)- $N^{\prime}$-(2,4,6-trimethoxybenzylidene) cyclopropanecarbohydrazide (5h): Off-white solid; M.p. $140-146^{\circ} \mathrm{C}$; Yield: $82 \%$; IR (KBr): $v_{\max } / \mathrm{cm}^{-1}$ 
3144 ( $\mathrm{NH}$ stretching), $1656(\mathrm{C}=\mathrm{O}$ stretching $), 1601$ $\left(\mathrm{C}=\mathrm{N}\right.$ stretching); ${ }^{1} \mathrm{H}$ NMR (400 MHz, DMSO- $\left.d_{6}\right) \delta$ $11.30(* 11.10, \mathrm{~s}, 1 \mathrm{H},-\mathrm{CO}-\mathrm{NH}-\mathrm{N}-), 8.26(* 8.17, \mathrm{~s}$, $1 \mathrm{H},-\mathrm{N}=\mathrm{CH}-), 7.49$ (d, $J=0.8 \mathrm{~Hz}, 1 \mathrm{H}, \mathrm{Ar}-\mathrm{H}), 6.36$ (dd, $J=2.0,3.2 \mathrm{~Hz}, 1 \mathrm{H}$, Ar-H), $6.26(\mathrm{~s}, 1 \mathrm{H}$, Ar-H), $6.24(\mathrm{~s}, 1 \mathrm{H}, \mathrm{Ar}-\mathrm{H}), 6.20(\mathrm{~d}, J=3.1 \mathrm{~Hz}, 1 \mathrm{H}, \mathrm{Ar}-\mathrm{H})$, $3.80\left(\mathrm{~s}, 3 \mathrm{H},-\mathrm{OCH}_{3}\right), 3.79\left(\mathrm{~s}, 3 \mathrm{H},-\mathrm{OCH}_{3}\right), 3.70(\mathrm{~s}, 3 \mathrm{H}$, $\left.-\mathrm{OCH}_{3}\right), 3.01-2.97$ ( $* 2.35-2.31, \mathrm{~m}, 1 \mathrm{H}$, cyc-CH-Ar), 2.39-2.36 (* 1.94-1.92, m, 1H, cyc-CH-CO-), 1.42-1.25 (m, 2H, cyc- $\left.\mathrm{CH}_{2}-\right) ;{ }^{13} \mathrm{C}$ NMR (100 MHz, DMSO- $d_{6}$ ) $\delta 171.9(\mathrm{C}=\mathrm{O}), 166.2,162(* 161.8), 159.7(* 159.7)$ (Ar-C), $154(* 153.8)(\mathrm{C}=\mathrm{N}), 141.7(* 141.3), 138.3$, 110.5, 105, 104.7 (* 104.0), 91.11 (* 91.10) (Ar-C), 55.8, 55.7, $55.3\left(\mathrm{OCH}_{3}\right), 22.3(* 19.5), 18.3(* 17.4)$, 13.7 (* 12.7); ESI-MS, $m / z: 345.2[\mathrm{M}-\mathrm{H}]^{+}$.

(E) - 2 - (furan - 2 - yl) - $N^{\prime}$ - (4-(methylthio) benzylidene) cyclopropanecarbohydrazide (5i): Off-white solid; M.p. $148-152^{\circ} \mathrm{C}$; Yield: $73 \mathrm{mg}$; 57\%; IR (KBr): $v_{\max } / \mathrm{cm}^{-1} 3250$ ( $\mathrm{NH}$ stretching), $1653(\mathrm{C}=\mathrm{O}$ stretching), 1599 ( $\mathrm{C}=\mathrm{N}$ stretching); ${ }^{1} \mathrm{H}$ NMR $(400 \mathrm{MHz}$, DMSO$\left.d_{6}\right) \delta 11.62(* 11.47, \mathrm{~s}, 1 \mathrm{H},-\mathrm{CO}-\mathrm{NH}-\mathrm{N}-), 8.0(* 7.98$, $\mathrm{s}, 1 \mathrm{H},-\mathrm{N}=\mathrm{CH}-), 7.61-7.59(\mathrm{~d}, J=8.5 \mathrm{~Hz}, 1 \mathrm{H}, \mathrm{Ar}-\mathrm{H})$, 7.56-7.54 (d, $J=8.5 \mathrm{~Hz}, 1 \mathrm{H}, \mathrm{Ar}-\mathrm{H}), 7.51-7.49(\mathrm{dd}$, $J=1.0,4.1, \mathrm{~Hz}, 1 \mathrm{H}, \mathrm{Ar}-\mathrm{H}), 7.30-7.26(\mathrm{t}, J=8.5 \mathrm{~Hz}$, $2 \mathrm{H}, \mathrm{Ar}-\mathrm{H}), 6.37-6.36$ (dd, $J=2.0,4.1 \mathrm{~Hz}, 1 \mathrm{H}, \mathrm{Ar}-\mathrm{H})$, 6.24-6.21 (dd, $J=3.1,7.0 \mathrm{~Hz}, 1 \mathrm{H}, \mathrm{Ar}-\mathrm{H}), 2.90-2.87$ (*2.37-2.33, m, 1H, cyc-CH-Ar), $2.52\left(\mathrm{~s}, 3 \mathrm{H},-\mathrm{SCH}_{3}\right)$, 2.46-2.42 (*1.98-1.95, m, 1H, cyc-CH-CO-), 1.43-1.30 (m, 2H, cyc- $\left.\mathrm{CH}_{2-}\right) ;{ }^{13} \mathrm{C}$ NMR (100 MHz, DMSO$\left.d_{6}\right) \delta 172.6(\mathrm{C}=\mathrm{O}), 167.4(\mathrm{Ar}-\mathrm{C}), 154.2(* 154.1)$ $(\mathrm{C}=\mathrm{N}), 146,143.5,141.9(* 141.8), 141.2(* 140.9)$, $131.1(* 130), 127.8$ (* 127.5), 126.2 (* 126.1), 111.1, 105.6 (Ar-C), $22.9\left(-\mathrm{SCH}_{3}\right), 19.8$ (* 18.8), 14.7 (* 14.5), 13.4 (Cyclopropane); ESI-MS, $m / z: 301.23$ $[\mathrm{M}+\mathrm{H}]^{+}$.

(E)-2-(furan-2-yl)-N'-(pyridin-4-ylmethylene) cyclopropanecarbohydrazide (5j): Yellow solid; M.p. 48$52^{\circ} \mathrm{C}$; Yield; $50 \mathrm{mg}$, 46\%; IR (KBr): $v_{\max } / \mathrm{cm}^{-1} 3196$ (NH stretching), $1667(\mathrm{C}=\mathrm{O}$ stretching), $1598(\mathrm{C}=\mathrm{N}$ stretching); ${ }^{1} \mathrm{H}$ NMR (400 MHz, DMSO- $\left.d_{6}\right) \delta 11.94$ (* 11.78, s, 1H, -CO-NH-N-), 8.67-8.58 (m, 2H, Ar-H), $8.14(* 8.02, \mathrm{~s}, 1 \mathrm{H},-\mathrm{N}=\mathrm{CH}-), 7.61-7.56(\mathrm{dd}, J=5.0$, $16 \mathrm{~Hz}, 2 \mathrm{H}, \mathrm{Ar}-\mathrm{H}), 7.51$ (d, $J=4.7 \mathrm{~Hz}, 1 \mathrm{H}, \mathrm{Ar}-\mathrm{H})$, 6.37 (s, $1 \mathrm{H}, \mathrm{Ar}-\mathrm{H}), 6.25-6.23(\mathrm{dd}, J=2.6,7.2 \mathrm{~Hz}, 1 \mathrm{H}$, $\mathrm{Ar}-\mathrm{H}), 2.95-2.90$ (* 2.42-2.38, m, 1H, cyc-CH-ArH), 2.49-2.46 (* 2.03-1.90, m, 1H, cyc-CH-Ar), 1.44-1.21 $\left(\mathrm{m}, 2 \mathrm{H}\right.$, cyc- $\left.\mathrm{CH}_{2}-\right) ;{ }^{13} \mathrm{C}$ NMR (100 MHz, DMSO- $\left.d_{6}\right)$ $\delta 172.6(\mathrm{C}=\mathrm{O}), 167.4(\mathrm{Ar}-\mathrm{C}), 153.5,153.4(\mathrm{C}=\mathrm{N})$, 150.17 (* 150.13), $143.5,141.5$ (* 141.4), 141.1 (*141.0), 120.8, (* 120.5), 110.6, 105.2 (Ar-C), 22.4
(* 19.2), 18.5 (* 18.1), 14.2 (* 13.1) (Cyclopropane); ESI-MS, $m / z: 256.2[\mathrm{M}+\mathrm{H}]^{+}$.

(E)-2-(furan-2-yl)-N'-(pyridin-2-ylmethylene) cyclopropanecarbohydrazide (5k): Off-white solid; Yield: 45 mg, 29\%; M.p. $150-156^{\circ} \mathrm{C}$; IR (KBr): $v_{\max } / \mathrm{cm}^{-1} 3183$ (NH stretching), $1661(\mathrm{C}=\mathrm{O}$ stretching), $1577(\mathrm{C}=\mathrm{N}$ stretching); ${ }^{1} \mathrm{H}$ NMR (400 MHz, DMSO- $\left.d_{6}\right) \delta 11.88$ (* 11.77, brs, $1 \mathrm{H},-\mathrm{CO}-\mathrm{NH}-\mathrm{N}-), 8.59-8.56(\mathrm{t}, J=5.0$ $\mathrm{Hz}, 1 \mathrm{H}, \mathrm{Ar}-\mathrm{H}), 8.15$ (* 8.08, s, $1 \mathrm{H},-\mathrm{N}=\mathrm{CH}-), 7.90-$ 7.81 (m, 2H, Ar-H), 7.51 (s, 1H, Ar-H), 7.40-7.35 (m, $1 \mathrm{H}, \mathrm{Ar}-\mathrm{H}), 6.37$ (d, $J=1.9 \mathrm{~Hz}, 1 \mathrm{H}, \mathrm{Ar}-\mathrm{H}), 6.26-6.24$ (dd, $J=3.1,6.2 \mathrm{~Hz}, 1 \mathrm{H}, \mathrm{Ar}-\mathrm{H}), 2.93-2.91$ (* 2.412.39, m, 1H, cyc-CH-Ar), 2.49 (* 1.99-1.97, m, 1H, cyc-CH-CO-), 1.45-1.34 (m, 2H, cyc- $\left.\mathrm{CH}_{2-}\right) ;{ }^{13} \mathrm{C}$ NMR $\left(100 \mathrm{MHz}, \mathrm{DMSO}-d_{6}\right) \delta 172.4(\mathrm{C}=\mathrm{O}), 167.3$ (Ar-C), $153.5(* 153.1)(\mathrm{C}=\mathrm{N}), 149.4,146.2,141.4(* 141.3)$, $136.7,124.2(* 124.0), 119.7(* 119.5), 110.6,105.2$ (Ar-C), $22.4(* 19.2), 18.5(* 18.0), 14.2(* 13.1)$ (Cyclopropane); ESI-MS, $m / z: 256.2[\mathrm{M}+\mathrm{H}]^{+}$.

(E)-2-(furan-2-yl)-N'-((6-methylpyridin-3-yl)methylene) cyclopropanecarbohydrazide (5l): White solid; Yield: $58 \mathrm{mg}, 51 \%$; M.p. $198-202^{\circ} \mathrm{C}$; IR (KBr): $v_{\max } / \mathrm{cm}^{-1}$ 3177 (NH stretching), 1688 ( $\mathrm{C}=\mathrm{O}$ stretching), 1605 $\left(\mathrm{C}=\mathrm{N}\right.$ stretching); ${ }^{1} \mathrm{H}$ NMR (400 MHz, DMSO- $\left.d_{6}\right) \delta$ 11.76 (* 11.60, s, 1H, -CO-NH-N-), 8.67 (* 8.62, d, $J=1.7 \mathrm{~Hz}, 1 \mathrm{H}, \mathrm{Ar}-\mathrm{H}), 8.17(* 8.04, \mathrm{~s}, 1 \mathrm{H},-\mathrm{N}=\mathrm{CH}-)$, 7.98-7.92 (m, 1H, Ar-H), 7.50 (s, 1H, Ar-H), 7.32-7.27 (q, $J=8.4 \mathrm{~Hz}, 1 \mathrm{H}, \mathrm{Ar}-\mathrm{H}), 6.38-6.36(\mathrm{dd}, J=1.8,3.0$ $\mathrm{Hz}, 1 \mathrm{H}, \mathrm{Ar}-\mathrm{H}), 6.25-6.22(\mathrm{dd}, J=3.2,6.2 \mathrm{~Hz}, 1 \mathrm{H}$, Ar-H), 2.93-2.90 (* 2.39-2.35, m, 1H, cyc-CH-Ar), 2.49 (s, 3H, Ar- $\left.\mathrm{CH}_{3}\right), 2.38$ * 1.99-1.97, m, 1H, cyc$\mathrm{CH}), 1.44-1.32\left(\mathrm{~m}, 2 \mathrm{H}\right.$, cyc- $\left.\mathrm{CH}_{2}-\right):{ }^{13} \mathrm{C}$ NMR (100 $\left.\mathrm{MHz}, \mathrm{DMSO}-d_{6}\right) 172.2(\mathrm{C}=\mathrm{O}), 167-(\mathrm{Ar}-\mathrm{C}), 153.6$ $(* 153.5)(\mathrm{C}=\mathrm{N}), 148(* 147.8), 143.4,141.4(* 141.3)$, $133.5(* 133.2), 127.3(* 127.2), 123.2,110.5,105.1$ (Ar-C), $23.9\left(\mathrm{Ar}-\mathrm{CH}_{3}\right), 22.3$ (* 19.2), 18.4 (* 17.8), 14.1 (* 12.9)-(Cyclopropane); ESI-MS, $m / z: 270.26$ $[\mathrm{M}+\mathrm{H}]^{+}$.

(E)-N'-((1H-indol-5-yl)methylene)-2-(furan-2-yl)cyclopropanecarbohydrazide $(\mathbf{5 m})$ : Off-white solid; Yield: $65 \mathrm{mg}$, 52\%; M.p. $260-264^{\circ} \mathrm{C}$; IR (KBr): $v_{\max } / \mathrm{cm}^{-1}$ 3190 ( $\mathrm{NH}$ stretching), 3141 (Indole-NH stretching), $1655(\mathrm{C}=\mathrm{O}$ stretching $), 1609(\mathrm{C}=\mathrm{N}$ stretching $) ;{ }^{1} \mathrm{H}$ NMR $\left(400 \mathrm{MHz}, \mathrm{DMSO}-d_{6}\right) \delta 11.81(\mathrm{~s}, 1 \mathrm{H}$, indole$\mathrm{NH}), 11.62(* 11.45, \mathrm{~s}, 1 \mathrm{H},-\mathrm{CO}-\mathrm{NH}-\mathrm{N}-), 8.51(* 8.47$, $\mathrm{d}, J=1.9 \mathrm{~Hz}, 1 \mathrm{H},-\mathrm{N}=\mathrm{CH}-), 8.27-8.15(\mathrm{~m}, 2 \mathrm{H}, \mathrm{Ar}-\mathrm{H})$, 7.52-7.50 (dd, $J=0.8,5.4 \mathrm{~Hz}, 2 \mathrm{H}, \mathrm{Ar}-\mathrm{H}), 6.52-6.49$ (m, 1H, Ar-H), 6.38-6.36 (dd, $J=3.0,5.1, \mathrm{~Hz}, 2 \mathrm{H}$, 
Ar-H), 6.26-6.22 (dd, $J=3.3,12.4 \mathrm{~Hz}, 1 \mathrm{H}$, Ar-H), 2.98-2.94 (* 2.39-2.34, m, 1H, сус-CH-Ar), 2.48 (* 1.99-1.97, m, 1H, cyc-CH-CO-), 1.44-1.30 (m, 2H, cyc- $\left.\mathrm{CH}_{2}-\right) ;{ }^{13} \mathrm{C}$ NMR $\left(100 \mathrm{MHz}, \mathrm{DMSO}-d_{6}\right) \delta 172.0$ $(\mathrm{C}=\mathrm{O}), 166.8$ (Ar-C), 153.8 (* 153.6), 149.1 (* 149), $145.4,142.8$ (* 142.4), 141.4 (* 141.1), 127.3, 126.2 (* 125.8), 122.28 (* 122.26), 119.4, 110.6, 105.1, 100.5 (Ar-C), 22.4 (* 19.3), 18.3 (* 17.6), 14 (* 12.9) (Cyclopropane); ESI-MS, $m / z: 292.9$ [M-H] $]^{+}$.

(E)- $N^{\prime}-((1 H$-indol-4-yl)methylene)-2-(furan-2-yl)cyclopropanecarbohydrazide (5n): Light yellow solid; Yield: $60 \mathrm{mg}$; 48\%; M.p. $152-156^{\circ} \mathrm{C}$; IR (KBr): $v_{\max } / \mathrm{cm}^{-1} 3411$ (Indole-NH stretching), $3181(\mathrm{NH}$ stretching $), 1663(\mathrm{C}=\mathrm{O}$ stretching $), 1611(\mathrm{C}=\mathrm{N}$ stretching); ${ }^{1} \mathrm{H}$ NMR (400 MHz, DMSO- $\left.d_{6}\right) \delta 11.6(* 11.41, \mathrm{~s}$, $1 \mathrm{H},-\mathrm{CO}-\mathrm{NH}-\mathrm{N}-), 11.32$ (*11.30, brs, $1 \mathrm{H}, \mathrm{Ar}-\mathrm{NH}), 8.36$ (* 8.27, s, $1 \mathrm{H},-\mathrm{N}=\mathrm{CH}-) 7.54(* 7.50, \mathrm{~d}, J=1.1 \mathrm{~Hz}$, 1H, Ar-H), 7.43-7.47 (m, 1H, Ar-H), 7.39-7.37 (*6.80$6.79, \mathrm{t}, J=2.7 \mathrm{~Hz}, 1 \mathrm{H}, \mathrm{Ar}-\mathrm{H}), 7.45(* 7.23-7.21, \mathrm{~d}$, $J=7 \mathrm{~Hz}, 1 \mathrm{H}, \mathrm{Ar}-\mathrm{H}), 7.15-7.05$ (m, 2H, Ar-H), 6.396.36 (m, 1H, Ar-H), 6.26-6.23 (dd, $J=3.1,9.2 \mathrm{~Hz}, 1 \mathrm{H}$, Ar-H), 3.01 (*2.40, m, 1H, cyc-CH-Ar), 2.43 (*2.01, $\mathrm{m}, 1 \mathrm{H}$, cyc-CH-CO-), 1.53-1.32 (m, $2 \mathrm{H}$, cyc- $\mathrm{CH}_{2}-$ ); ${ }^{13} \mathrm{C}$ NMR (100 MHz, DMSO- $\left.d_{6}\right) \delta 171.9(\mathrm{C}=\mathrm{O})$, 166.7 (Ar-C), $153.74(* 153.7)(\mathrm{C}=\mathrm{N}), 147.1,145.2$, 141.4 (* 141.3), 126.5 (* 126.4), 125.3 (* 125.2), 1212.4 (* 121.2), 120.6, $113.5(* 113.4), 110.6,105.1$, 102.2, 101.5 (Ar-C), 22.4 (* 19.6), 18.4 (* 17.6), 13.5 (* 12.9) (Cyclopropane); ESI-MS, $m / z: 294.0$ $[\mathrm{M}+\mathrm{H}]^{+}$.

(E)-N'-((1-ethyl-1H-pyrazol-5-yl)methylene)-2-(furan2-yl)cyclopropanecarbohydrazide (5o): Off-white solid; Yield: $62 \mathrm{mg}$, 54\%; M.p. 116-120 C; IR (KBr): $v_{\max } / \mathrm{cm}^{-1} 3190(\mathrm{NH}$ stretching), $1663(\mathrm{C}=\mathrm{O}$ stretching), $1621\left(\mathrm{C}=\mathrm{N}\right.$ stretching); ${ }^{1} \mathrm{H}$ NMR $(400 \mathrm{MHz}$, DMSO- $\left.d_{6}\right) \delta 11.73$ (* 11.54, s, $\left.1 \mathrm{H},-\mathrm{CO}-\mathrm{NH}-\mathrm{N}-\right)$, $8.21(* 8.07, \mathrm{~s}, 1 \mathrm{H},-\mathrm{CO}-\mathrm{NH}-\mathrm{N}-), 7.49$ (d, $J=1.6$ $\mathrm{Hz}, 1 \mathrm{H}, \mathrm{Ar}-\mathrm{H}), 7.46-7.43(\mathrm{dd}, J=9.0,20.9 \mathrm{~Hz}, 1 \mathrm{H}$, Ar-H), 6.59-6.56 (dd, $J=3.0,11.3 \mathrm{~Hz}, 1 \mathrm{H}$, Ar-H), 6.37-6.35 (dd, $J=3.2,5.0 \mathrm{~Hz}, 1 \mathrm{H}, \mathrm{Ar}-\mathrm{H}), 6.23-6.21$ $(\mathrm{t}, J=3.5 \mathrm{~Hz}, 1 \mathrm{H}$, Ar-H), 4.41-4.35 (* 4.33-4.23, m, $\left.2 \mathrm{H},-\mathrm{N}-\mathrm{CH}_{2}-\right), 2.77-2.74$ (*2.49, m, 1H, cyc-CH-ArH), 2.47 (* 1.96, m, 1H, cyc-CH-CO-), 1.50-1.36 (m, 2H, cyc- $\left.\mathrm{CH}_{2}-\right), 1.30\left(* 1.16, \mathrm{t}, J=2.3 \mathrm{~Hz}, 3 \mathrm{H},-\mathrm{CH}_{3}\right)$ : ${ }^{13} \mathrm{C}$ NMR (100 MHz, DMSO- $\left.d_{6}\right) \delta 172.1(\mathrm{C}=\mathrm{O}), 167$ $($ Ar-C), $153.6(* 153.5)(\mathrm{C}=\mathrm{N}), 141.3,138.2(* 138.1)$, 135.9 (* 135.4), 133.3, 110.6, 108.5, 107.8, 105.2 (* 105) (Ar-C), 45.7 (* 45.4) $\left(\mathrm{CH}_{2}-\right), 22.4(* 19.6)$, $18.5(* 17.8)$ (Cyclopropane), $15.4(* 15)-\left(\mathrm{CH}_{3}\right) 13.5$ (* 13) (Cyclopropane); ESI-MS, $m / z: 273.2[\mathrm{M}+\mathrm{H}]^{+}$.
(E)-N'-((1H-imidazol-2-yl)methylene $)-2-($ furan-2-yl) cyclopropanecarbohydrazide (5p): Off-white solid; M.p. $242-246^{\circ} \mathrm{C}$; Yield: $70 \mathrm{mg}, 68 \%$; IR (KBr): $v_{\max } / \mathrm{cm}^{-1} 3251 \quad$ (Imidazole-NH stretching), 3173 (NH stretching), $1661(\mathrm{C}=\mathrm{O}$ stretching $), 1611(\mathrm{C}=\mathrm{N}$ stretching); ${ }^{1} \mathrm{H}$ NMR (400 MHz, DMSO- $\left.d_{6}\right) \delta 13.3$ (* 12.6, s, 1H, Ar-NH), 11.68 (*11.48, s, $1 \mathrm{H},-\mathrm{CO}-$ NH-N-), 8.05 (* 7.9, s, 1H, -N=CH-), 7.50 (s, 1H, Ar$\mathrm{H})$, 7.29-7.11 (m, 2H, Ar-H), 6.37-6.36 (dd, $J=1.9$, $2.7 \mathrm{~Hz}, 1 \mathrm{H}, \mathrm{Ar}-\mathrm{H}), 6.25-6.23(\mathrm{dd}, J=3.0,7.0 \mathrm{~Hz}, 1 \mathrm{H}$, Ar-H), 3.09-3.06 (* 2.41-2.36, m, 1H, cyc-CH-Ar), 2.49-2.44 (* 1.97-1.93, m, 1H, cyc-CH-CO), 1.44-1.32 $\left(\mathrm{m}, 2 \mathrm{H}\right.$, cyc- $\left.\mathrm{CH}_{2}-\right) ;{ }^{13} \mathrm{C}$ NMR (100 MHz, DMSO- $\left.d_{6}\right)$ $\delta 172.4(\mathrm{C}=\mathrm{O}), 167(\mathrm{Ar}-\mathrm{C}), 153.7(* 153.5)(\mathrm{C}=\mathrm{N})$, 142.4 (* 142.2), $141.4(* 141.3), 137.9,135,110.6$ (* 110.5), 105.3 (* 105.2) (Ar-C), 22.5 (* 18.8), 18.4 (* 17.8), $14.3(* 13)$ (Cyclopropane); ESI-MS, $m / z$ : $245.2[\mathrm{M}+\mathrm{H}]^{+}$.

(E)-N' -((1H-pyrrol-2-yl)methylene $)$-2 - (furan-2-yl) cyclopropanecarbohydrazide $(\mathbf{5 q})$ : Light brown solid; M.p. 206- $210^{\circ} \mathrm{C}$; Yield: $43 \mathrm{mg}, 42.1 \%$; IR (KBr): $v_{\max } / \mathrm{cm}^{-1} 3208$ (NH stretching), 3148 (Pyrrole-NH stretching $), 1643(\mathrm{C}=\mathrm{O}$ stretching $), 1610(\mathrm{C}=\mathrm{N}$ stretching); ${ }^{1} \mathrm{H}$ NMR (400 MHz, DMSO- $\left.d_{6}\right) \delta 11.43(* 11.35 \mathrm{~s}$, 1H, Ar-NH), 11.32 (*11.16, s, 1H, -CO-NH-N-), 7.99 (* 7.86, s, $1 \mathrm{H},-\mathrm{N}=\mathrm{CH}-), 7.49$ (s, $1 \mathrm{H}$, Ar-H), 6.87 (s, 1H, Ar-H), 6.43 (* 6.37, s, 1H, Ar-H), 6.36 (d, $J=1.5 \mathrm{~Hz}, 1 \mathrm{H}, \mathrm{Ar}-\mathrm{H}), 6.22(\mathrm{dd}, J=3.0,9.0, \mathrm{~Hz}, 1 \mathrm{H}$, Ar-H), 6.09-6.07 (m, 1H, Ar-H), 3.07-3.02 (* 2.362.31, m, 1H, cyc-CH-Ar), 2.49-2.41 (* 1.96-1.91, m, $1 \mathrm{H}$, cyc-CH-CO-), $1.39-1.29$ (m, $2 \mathrm{H}$, cyc- $\left.\mathrm{CH}_{2-}\right) ;{ }^{13} \mathrm{C}$ NMR (100 MHz, DMSO-d $)_{6} \delta 171.9(\mathrm{C}=\mathrm{O}), 166.4$ (Ar-C), $153.9(* 153.7)(\mathrm{C}=\mathrm{N}), 141.4(* 141.3), 136.3$, 127 (* 126.8), $122.3(* 121.7), 113.1,109.1(* 109)$, $105.2(* 105.1)$ (Ar-C), $22.5(* 18.9), 18.2(* 17.5)$, 14.1 * 12.8) (Cyclopropane) ESI-MS, $\mathrm{m} / z: 242.0$ $[\mathrm{M}-\mathrm{H}]^{+}$.

(E)-2-(furan-2-yl)- $N^{\prime}$-(thiophen-2-ylmethylene $)$ cyclopropanecarbohydrazide $(\mathbf{5} \boldsymbol{r})$ : Off-white solid; Yield: $120 \mathrm{mg}, 76 \%$; M.p. $170-174^{\circ} \mathrm{C}$; IR (KBr): $v_{\max } / \mathrm{cm}^{-1}$ 3205 (NH stretching), 1651 ( $\mathrm{C}=\mathrm{O}$ stretching), 1595 $\left(\mathrm{C}=\mathrm{N}\right.$ stretching); ${ }^{1} \mathrm{H}$ NMR (400 MHz, DMSO- $\left.d_{6}\right) \delta$ $11.61(* 11.42, \mathrm{~s}, 1 \mathrm{H},-\mathrm{CO}-\mathrm{NH}-\mathrm{N}-), 8.36(* 8.20, \mathrm{~s}$, $1 \mathrm{H},-\mathrm{N}=\mathrm{CH}-), 7.62(* 7.58, \mathrm{~d}, J=4.9 \mathrm{~Hz}, 1 \mathrm{H}, \mathrm{Ar}-\mathrm{H})$, $7.57(\mathrm{~d}, J=5.1 \mathrm{~Hz}, 1 \mathrm{H}$, Ar-H), 7.51-7.50 (* $7.49 \mathrm{dd}$, $J=0.8,1.8 \mathrm{~Hz}, 1 \mathrm{H}$, Ar-H), 7.12-7.10 (m, 1H, Ar-H), 6.37-6.36 (d, $J=1.8 \mathrm{~Hz}, \quad 1 \mathrm{H}, \quad$ Ar-H), $6.23-6.22$ (t, $J=3.2 \mathrm{~Hz}, 1 \mathrm{H}, \mathrm{Ar}-\mathrm{H}$ ), 2.80-2.77 (*2.38-2.33, m, 1H, cyc-CH-Ar), 2.49-2.48 (* 1.97-1.92, m, 1H, cyc-CHCO- $), 1.45-1.29\left(\mathrm{~m}, 2 \mathrm{H}\right.$, cyc- $\left.\mathrm{CH}_{2}-\right) ;{ }^{13} \mathrm{C}$ NMR (100 
MHz, DMSO- $\left.d_{6}\right) \delta 171.9(\mathrm{C}=\mathrm{O}), 166.9$ (Ar-C), 153.6 $(* 153.5)(\mathrm{C}=\mathrm{N}), 141.4(* 141.3), 138.9(* 138.4)$, 130.6, $128.6(* 128), 127.7(* 127.6), 110.6(* 110.5)$, 105.1 (Ar-C), $22.4(* 19.1), 18.4(* 17.8), 14(* 12.9)$ (Cyclopropane); ESI-MS, $m / z: 261.1[\mathrm{M}+\mathrm{H}]^{+}$.

(E)-2-(furan-2-yl)-N'-(furan-3-ylmethylene) cyclopropanecarbohydrazide (5s): Brown solid; Yield: 43 mg, 42\%; M.p. $170-174^{\circ} \mathrm{C}$; IR (KBr): $v_{\max } / \mathrm{cm}^{-1} 3207$ (NH stretching), $1653(\mathrm{C}=\mathrm{O}$ stretching $), 1625(\mathrm{C}=\mathrm{N}$ stretching); ${ }^{1} \mathrm{H}$ NMR (400 MHz, DMSO- $\left.d_{6}\right) \delta 11.51$ (* 11.38, s, $1 \mathrm{H},-\mathrm{CO}-\mathrm{NH}-\mathrm{N}-), 8.01(* 7.98 \mathrm{~s}, 1 \mathrm{H}$, $-\mathrm{N}=\mathrm{CH}-), 8.07-7.98$ (d, $J=6.5 \mathrm{~Hz}, 1 \mathrm{H}, \mathrm{Ar}-\mathrm{H}), 7.72-$ 7.69 (d, $J=11.2 \mathrm{~Hz}, 1 \mathrm{H}$, Ar-H), 7.49 (s, 1H, Ar-H), 6.72-6.70 (d, $J=9.4 \mathrm{~Hz}, 1 \mathrm{H}, \operatorname{Ar}-\mathrm{H}), 6.37-6.31(\mathrm{~d}$, $J=2 \mathrm{~Hz}, 1 \mathrm{H}, \mathrm{Ar}-\mathrm{H}), 6.22(\mathrm{t}, J=3.3 \mathrm{~Hz}, 1 \mathrm{H}, \mathrm{Ar}-\mathrm{H})$, 2.86-2.84 (* 2.35-2.31, m, 1H, cyc-CH-Ar), 2.45-2.41 (* 1.95-1.93, m, 1H, cyc-CH-CO-), 1.41-1.30 (m, 2H, cyc- $\left.\mathrm{CH}_{2-}\right) ;{ }^{13} \mathrm{C}$ NMR $\left(100 \mathrm{MHz}, \mathrm{DMSO}-d_{6}\right) \delta 172.0$ $(\mathrm{C}=\mathrm{O}), 166.7(\mathrm{Ar}-\mathrm{C}), 153.7(* 153.6)(\mathrm{C}=\mathrm{N}), 144.7$ (* 144.6), 141.4 (* 141.3), 136.9, 122.4 (* 122.3), 110.6, 107.1 (* 106.9), 105.1 (Ar-C) 22.3 (* 19.1), 18.3 (* 17.6), $14(* 12.8)$ (Cyclopropane); ESI-MS, $m / z$ : $245.18[\mathrm{M}+\mathrm{H}]^{+}$.

(E)-2-(furan-2-yl)- $N^{\prime}-((1-$ methyl-1H-pyrazol-4-yl) methylene)cyclopropanecarbohydrazide (5t): Light yellow solid; Yield: $120 \mathrm{mg}, 76 \%$; M.p. $170-174^{\circ} \mathrm{C}$; IR (KBr): $v_{\max } / \mathrm{cm}^{-1} 3213$ (NH stretching), 1652 (C=Ostretching), $1618\left(\mathrm{C}=\mathrm{N}\right.$ stretching); ${ }^{1} \mathrm{H}$ NMR (400 $\left.\mathrm{MHz}, \mathrm{DMSO}-d_{6}\right) \delta 11.36(* 11.2, \mathrm{~s}, 1 \mathrm{H},-\mathrm{CO}-\mathrm{NH}-)$, $8.03(* 7.92, \mathrm{~s}, 1 \mathrm{H},-\mathrm{N}=\mathrm{CH}-), 8.02(\mathrm{~s}, 1 \mathrm{H}, \mathrm{Ar}-\mathrm{H})$, 7.69-7.67 (d, $J=8.4 \mathrm{~Hz}, 1 \mathrm{H}, \operatorname{Ar}-\mathrm{H}), 7.49(\mathrm{~s}, 1 \mathrm{H}$, Ar-H), 6.36 (s, 1H, Ar-H), 6.22-6.20 (dd, $J=3.0,6.2$ $\mathrm{Hz}, 1 \mathrm{H}, \mathrm{Ar}-\mathrm{H}), 3.83$ (*3.81, s, $\left.3 \mathrm{H}, \mathrm{N}-\mathrm{CH}_{3}\right), 2.85-2.81$ (* 2.35-2.30, m, 1H, cyc-CH-Ar), 2.44-2.40 (* 1.94$1.90, \mathrm{~m}, 1 \mathrm{H}$, cyc-CH-CO-), $1.38-1.26$ (m, $2 \mathrm{H}$, cyc- $\mathrm{CH}_{2}-$ ); ${ }^{13} \mathrm{C}$ NMR (100 MHz, DMSO- $\left.d_{6}\right) \delta 171.6(\mathrm{C}=\mathrm{O})$, 166.4 (Ar-C), $153.8(* 153.6)(\mathrm{C}=\mathrm{N}), 141.3(* 141.2)$, 137.5 (* 137.5), $130.6(* 130.2), 117.3,110.5,105.06$; (* 105.02) (Ar-C), 22.3 (* 19.1), 18.1 (* 17.4), 13.9 $(* 12.7)$ (Cyclopropane); ESI-MS, $m / z: 259.21[\mathrm{M}+\mathrm{H}]^{+}$.

\section{$*$ indicates the presence of isomer}

\subsection{Cytotoxicity assay}

The cytotoxicity of the compounds was determined on the basis of measurement of in vitro growth inhibition of tumor cell lines in 96 well plates by cell-mediated reduction of tetrazolium salt to water insoluble formazan crystals using 5-Fluorouracil as a standard. The cytotoxicity was assessed against a panel of four different human tumor cell lines: HeLa derived from human cervical cancer cells (ATCC No. CCL-2), MDAMB-231 derived from human breast adenocarcinoma cells (ATCC No. HTB-26), MCF7 derived from human breast adenocarcinoma cells (ATCC No HTB-22), A549 derived from human alveolar adenocarcinoma epithelial cells (ATCC No. CCL-185), and HEK-293 derived from normal human embryonic kidney cells (ATCC No. CRL-1573) using the MTT assay. ${ }^{23}$ The IC50 values (50\% inhibitory concentration) were calculated from the plotted absorbance data for the doseresponse curves. $\mathrm{IC}_{50}$ values (in $\mu \mathrm{M}$ ) are expressed as the average of three independent experiments.

\section{Results and Discussion}

\subsection{Synthesis and Characterization}

The synthetic sequence for the preparation of $(E)-\mathrm{N}^{\prime}-$ (substituted-benzylidene)-2-(furan-2-yl)cyclopropane carbohydrazide derivatives is presented in scheme 1 . The Knoevenagel condensation of 2-furfuraldehyde with malonic acid in presence of piperidine in pyridine gave acrylic acid (1). Esterification of $(E)$-3-(furan2-yl)acrylic acid (1), followed by Corey-Chaykovsky cyclopropanation reaction of ethyl 3-(furan-2-yl)acrylate (2) with trimethyl sulphoxonium iodide in presence of sodium hydride in DMSO at room temperature for $1 \mathrm{~h}$ produced ethyl 2-(furan-2-yl)cyclopropanecarboxylate (3). Hydrazinolysis of $\mathbf{3}$ in presence of hydrazine hydrate in 1,4-dioxane at $100^{\circ} \mathrm{C}$ for $24 \mathrm{~h}$ yielded 2(furan-2-yl)cyclopropanecarbohydrazide (4). Condensation of 2-(furan-2-yl)cyclopropanecarbohydrazide (4) with heteroaryl/aryl aldehydes (a-t) in presence of catalytic amount of conc. $\mathrm{HCl}$ resulted in substituted N- hetero/arylidene-2-(furan-2-yl) cyclopropane carbohydrazides (5a-t). In addition to the above reaction conditions, some greener reaction conditions viz., L-tyrosine, PEG-400, PEG-600, ZnO NPs and microwave were also tried. The details of the reaction conditions are given under experimental section.

Among the reaction conditions attempted, $\mathrm{ZnO} N \mathrm{NP}$ exhibited superior results in terms of high efficiency of reaction yields and purity of the isolated hydrazone derivatives 5a-t. All the newly synthesized compounds were sufficiently characterized by ${ }^{1} \mathrm{H} \mathrm{NMR},{ }^{13} \mathrm{C} \mathrm{NMR}$, mass and IR spectroscopic techniques. Moreover, all the compounds were found to exist as a mixture of two rotameric forms in solution, ${ }^{24}$ indicating the possibility of equilibrium and interconversion between rotamers (and/or configurational isomers) as indicated by their ${ }^{1} \mathrm{H}$ NMR spectra. Two sets of signals were observed 

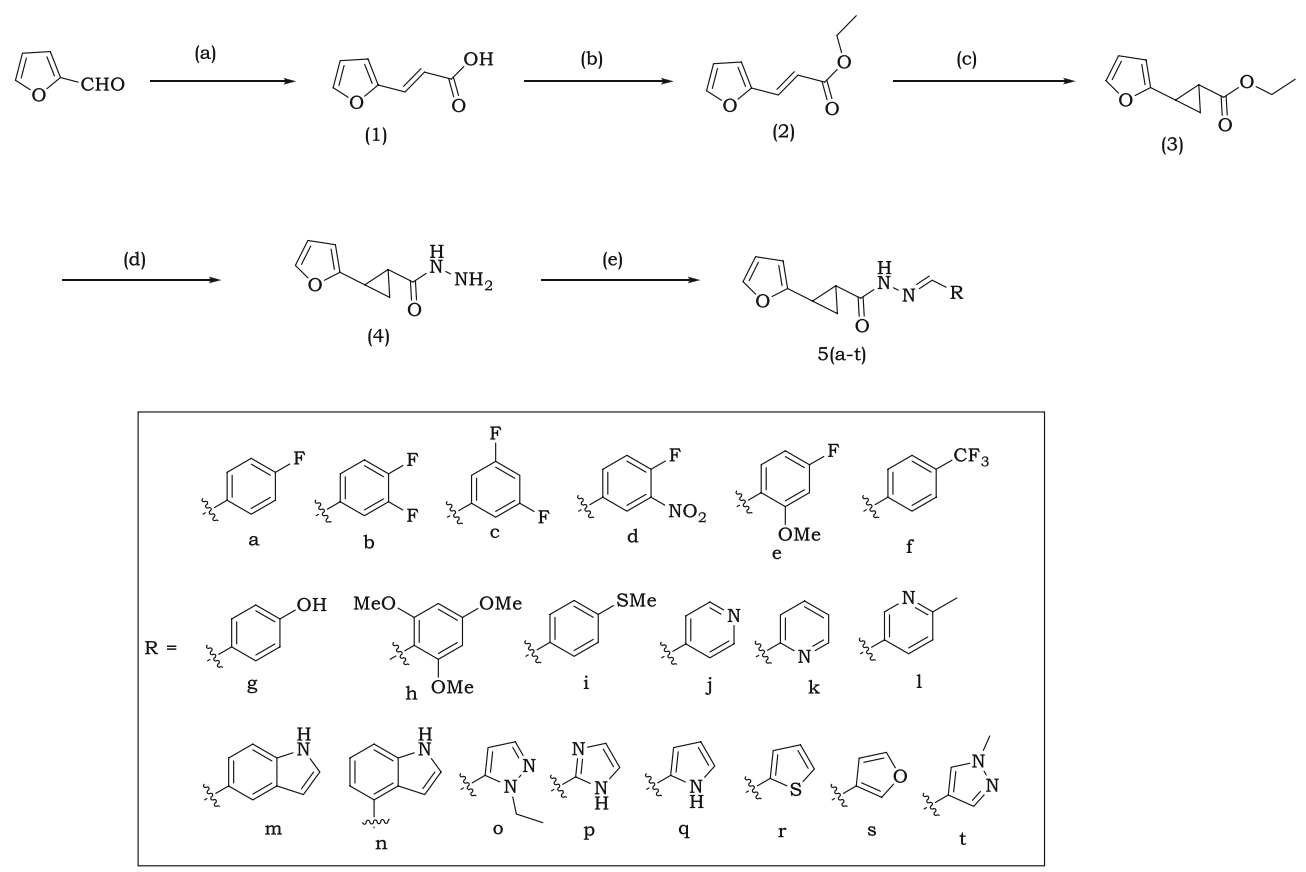

Scheme 1. (E)-N'-(substituted-benzylidene)-2-(furan-2-yl) cyclopropane carbohydrazide derivatives 5a-t. Reagents and conditions: a) Malonic acid, piperidine, in pyridine, $110^{\circ} \mathrm{C}$, $2 \mathrm{~h}$; b) Cat- $\mathrm{H}_{2} \mathrm{SO}_{4}$, ethanol, reflux, $16 \mathrm{~h}$; c) $\mathrm{NaH}$, Trimethyl sulfoxonium iodide, in DMSO, $\mathrm{rt}, 1 \mathrm{~h}$; d) Hydrazine hydrate, in 1,4-dioxane, $100^{\circ} \mathrm{C}, 24 \mathrm{~h}$; e) R-CHO (R = a-t), Zno NP, ethanol, room temperature, $30 \mathrm{~min}$.

for the groups $-\mathrm{CO}-\mathbf{N H}-\mathrm{N}-,-\mathrm{N}=\mathrm{CH}-$, cyclopropane ring, and the protons of aromatic and hetero aromatic rings that are in close proximity of the imine group $(-\mathrm{N}=\mathrm{CH}-)$.

As an example, the ${ }^{1} \mathrm{H}$ NMR of (E)-2-(furan-2yl)-N'-(2,4,6-trimethoxybenzylidene)cyclopropane carbohydrazide $\mathbf{5 h}$ is discussed here. A set of proton signals appearing at $11.30 \mathrm{ppm}$ and $11.10 \mathrm{ppm}$ corresponded to -CO-NH-N- group, while another set of proton signals resonating at $8.26 \mathrm{ppm}$ and $8.17 \mathrm{ppm}$ corresponded to $-\mathrm{N}=\mathrm{CH}$ - group. ${ }^{24}$ The proton signals resonating at $6.24 \mathrm{ppm}$ and $6.26 \mathrm{ppm}$ as singlet corresponded to the 2,4,6-trimethoxy phenyl ring. The doublet and doublet of doublets signals at $7.49 \mathrm{ppm}$, $6.36 \mathrm{ppm}$ and $6.20 \mathrm{ppm}$ corresponded to the furan ring. The multiplets appearing in the region 3.01 to $1.25 \mathrm{ppm}$ corresponded to the cyclopropane ring. ESI-MS spectrum carrying a base peak at $m / z 345.2[\mathrm{M}+1]^{+}$was also in agreement with the formation of compound $\mathbf{5 h}$. The IR stretching frequencies of all the compounds were found to be in the expected region.

\subsection{Cytotoxic activity}

Compounds 5a-t were screened for their cytotoxic potential against a panel of four human cancer cell lines such as HeLa, (Cervical cancer, ATCC No. CCL-2),
MDA-MB-231 (Breast cancer, ATCC No. HTB-26), MCF7 (Breast cancer, ATCC No. HTB-22), A549 (Lung cancer, ATCC No. CCL-185) and HEK-293 (Normal human embryonic kidney cells, ATCC No. CRL-1573) using the MTT assay. ${ }^{23}$ Among all the compounds screened (table 1), compounds $\mathbf{5 a}, \mathbf{5 l}, \mathbf{5 r}$ and $\mathbf{5 s}$ showed promising activity at micromolar concentration against all the tested cell lines with $\mathrm{IC}_{50}$ values ranging between 1.9-8.45 $\mu \mathrm{M}$. While some compounds such as 5c, 5f, 5g, 5h, 5m, 5n, 5q and $5 \mathrm{t}\left(\mathrm{IC}_{50}\right.$ values between 2.19-11.19 $\mu \mathrm{M}$ ) were quite promising and showed cytotoxicity selectively on either of the tested cell lines. Some compounds such as $\mathbf{5 b}, \mathbf{5 e}$, $\mathbf{5 j}, \mathbf{5 k}, \mathbf{5 o}$ and $\mathbf{5 p}$ showed no cytotoxicity against all the tested cell lines. Further, it was interesting to note that all the tested compounds showed no cytotoxicity against HEK-293. From a structure-activity relationship (SAR) perspective, these compounds have various aromatic rings (bearing mono fluoro, difluoro and trifluoromethyl substituent) and hetero aromatic rings (bearing five and six membered ring size with $\mathrm{N}, \mathrm{O}$ and $\mathrm{S}$ atom) attached to the basic cyclopropane carbohydrazide scaffold which might be contributing to these cytotoxic activities. The structure-activity relationship studies revealed that the cyclopropane carbohydrazide derivatives 5a $(\mathrm{R}=4$-fluoro phenyl), $5 \mathbf{l}(\mathrm{R}=2$-methyl pyridine), $5 \mathbf{r}(\mathrm{R}=$ thiophene $)$ and $\mathbf{5} \mathbf{s}(\mathrm{R}=$ furan $)$ 
Table 1. In vitro cytotoxicity of cyclopropane carbohydrazide derivatives.

\begin{tabular}{|c|c|c|c|c|c|}
\hline \multirow[t]{2}{*}{ Compd. } & \multicolumn{5}{|c|}{$\mathbf{I C}_{\mathbf{5 0}}(\mu \mathbf{M})$} \\
\hline & HeLa & MDA-MB-231 & MCF-7 & A549 & HEK 293 \\
\hline $5 \mathbf{a}$ & $5.57 \pm 0.33$ & $3.28 \pm 0.33$ & $3.09 \pm 0.35$ & $2.9 \pm 0.29$ & $-^{\mathrm{a}}$ \\
\hline $5 b$ & - & - & - & - & - \\
\hline $5 c$ & $6.86 \pm 0.28$ & $9.88 \pm 0.29$ & - & - & - \\
\hline $5 d$ & $11.9 \pm 0.35$ & $12.1 \pm 0.35$ & $19.5 \pm 0.28$ & $15.4 \pm 0.36$ & - \\
\hline $5 e$ & - & - & - & - & - \\
\hline $5 f$ & - & - & $8.6 \pm 0.35$ & - & - \\
\hline $5 \mathrm{~g}$ & $3.5 \pm 0.2$ & - & - & - & - \\
\hline $5 \mathrm{~h}$ & $4.6 \pm 0.29$ & - & - & - & - \\
\hline $5 \mathbf{i}$ & $14.25 \pm 0.41$ & $11.47 \pm 0.42$ & $19.82 \pm 0.34$ & $15.09 \pm 0.37$ & - \\
\hline $5 \mathbf{j}$ & - & - & - & - & - \\
\hline $5 \mathbf{k}$ & - & - & - & - & - \\
\hline 51 & $3.36 \pm 0.35$ & $4.87 \pm 0.4$ & $3.43 \pm 0.32$ & $5.26 \pm 0.35$ & - \\
\hline $5 \mathrm{~m}$ & - & - & $2.19 \pm 0.42$ & $4.69 \pm 0.39$ & - \\
\hline $5 n$ & $2.89 \pm 0.44$ & $5.32 \pm 0.43$ & $3.95 \pm 0.43$ & - & - \\
\hline 50 & - & - & - & - & - \\
\hline $5 p$ & - & - & - & - & - \\
\hline $5 q$ & - & - & - & $4.36 \pm 0.39$ & - \\
\hline $5 \mathrm{r}$ & $1.9 \pm 0.24$ & $2.9 \pm 0.33$ & $4.9 \pm 0.33$ & $8.45 \pm 0.35$ & - \\
\hline $5 s$ & $2.9 \pm 0.29$ & $2.47 \pm 0.42$ & $8.2 \pm 0.37$ & $7.09 \pm 0.41$ & _- \\
\hline $5 t$ & - & $11.19 \pm 0.49$ & $7.49 \pm 0.35$ & $25.1 \pm 0.89$ & - \\
\hline DOX & $0.36 \pm 0.14$ & $0.47 \pm 0.4$ & $0.98 \pm 0.14$ & $0.89 \pm 0.26$ & - \\
\hline
\end{tabular}

${ }^{a}$ No activity

showed more promising cytotoxicity. The molecules further validated using the molecular docking studies.

\subsection{Molecular docking studies}

The binding modes of the most active four compounds were determined at the colchicine binding site on the tubulin. The study was performed using extra precision (XP) mode in the GLIDE module ${ }^{25}$ of the Schrodinger suite. Before going to the docking studies, both the protein and ligands were to be prepared. With the protein preparation wizard, the retrieved protein from the protein data bank (PDB ID: 1SA0) was prepared by adding hydrogens, capping, removing unwanted water molecules, optimization and minimization using the force field OPLS-2005. The four finally selected compounds were prepared using the LigPrep module. ${ }^{26}$ Using the receptor grid generation from the Glide module, the protein active pocket was fixed and the prepared molecules were docked to the centroid. Additionally the molecules ADME properties were also checked using the QikProp application ${ }^{27}$ and furthermore the free binding energy of the complexes was also calculated using the Prime module.

In general, tubulin has vinca binding site, colchicine binding site and taxane binding site. Out of the three, several anticancer compounds of both natural and synthetic origin are supposed to bind in the colchicine binding site. ${ }^{28}$ In this study, we also performed the docking studies in this colchicine binding site. Especially the binding mode of Cys 241 residue binding mode with the compounds was analyzed as it is a crucial amino acid in the pocket. The majority of the binding pocket nature was hydrophobic due to the presence of residues Val, Cys, Ala, Pro, Leu, Met and Ile. Other residues like Asn and Thr made the pocket polar and Lys as positively charged. The four compounds have the common core $(E)-\mathrm{N}^{\prime}$-ethylidene-2-(furan-2-yl) cyclopropane carbohydrazide moiety with attached $\mathrm{R}$ group as depicted in the scheme 1. All the final four molecules made hydrogen bond with the important amino Cys 241 with varied distances and the also the number (figure 1). Compounds $\mathbf{5 s}$ and $5 \mathrm{r}$ displayed two hydrogen bonds, one with the keto group of the moiety $(\mathbf{5 s}-2.23 \AA$ and $\mathbf{5 r}-2.43 \AA)$ and another with the Nmethylene methanamine of the core moiety with almost nearly equal distance ( 2.13 and $2.10 \AA)$. Whereas in the 51 an 5 a compounds only one hydrogen bond (2.08 and $2.28 \AA$ ) was observed with the keto group of the moiety. Three molecules $\mathbf{5 s}, \mathbf{5 r}$ and $\mathbf{5 a}$ showed similar orientation i.e., functional group facing towards the Cys 241. This was reverse in the compound 5l, core moiety facing towards the Cys 241 which made the compound to form only single hydrogen. 1-methyl-2methylenehydrazine group of the compounds was the crucial part in producing the hydrogen bonds. 


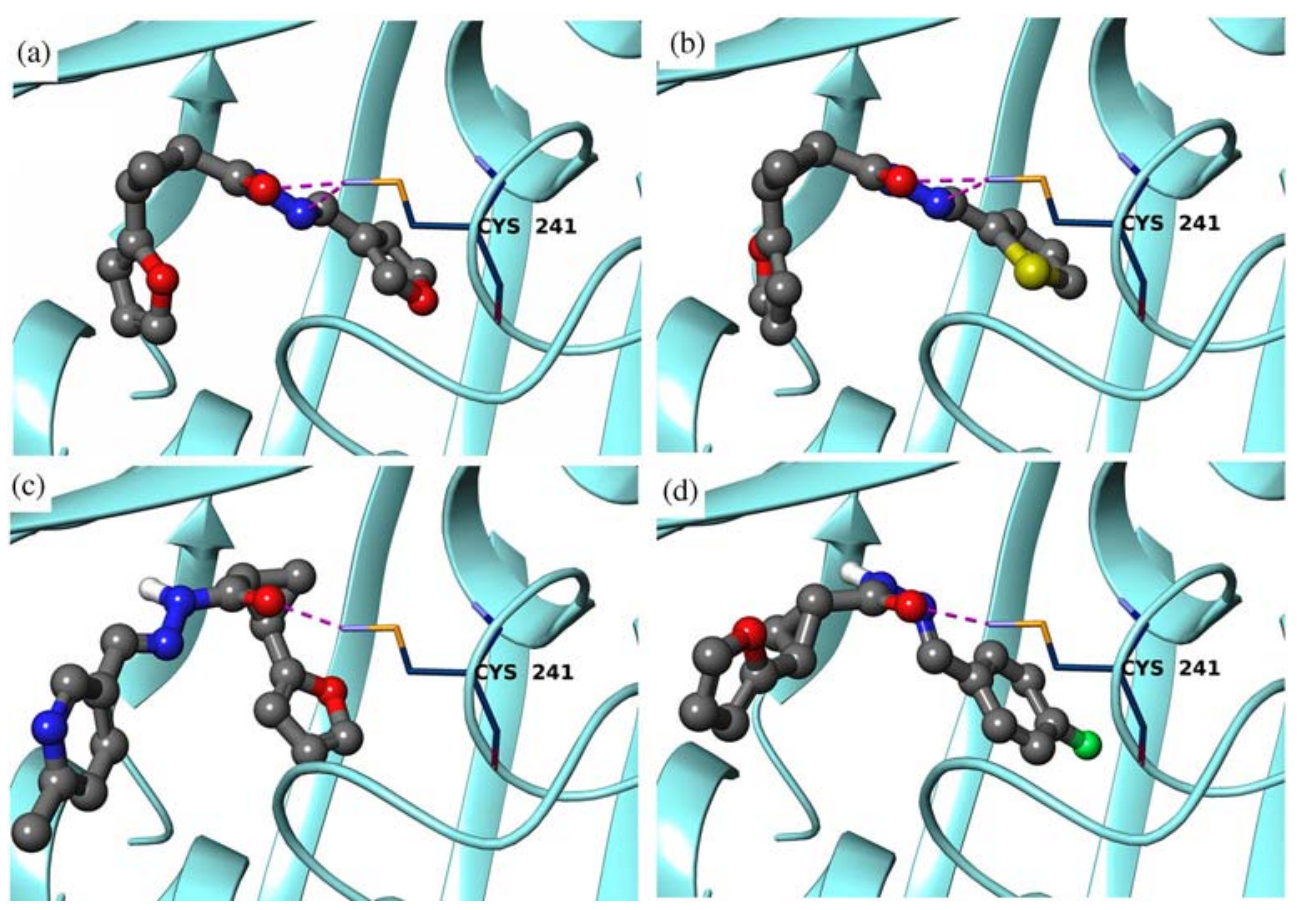

Figure 1. Binding modes of the compounds with Tubulin a) Compound $\mathbf{5 s}$ b) Compound $\mathbf{5 r}$ c) Compound $\mathbf{5 l}$ d) Compound $\mathbf{5 a}$.

The G-scores of the compounds $\mathbf{5 s}, \mathbf{5 r}, \mathbf{5 l}, \mathbf{5 a}$ are $-5.597,-5.543,-5.523,-5.499$. Coming to the fitness of the molecules into the pocket, all the four compounds were deeply fitted into the groove of the pocket as displayed in the figure 2 . The physiochemical properties were also calculated and tabulated in the table 2 . It was observed that four compounds exhibited good human oral absorption and lipinski's rule of five. Apart from the docking studies, the binding free energies of the four complexes were calculated (table 2). Among the four, 5r showed highest and 5a showed lowest binding free energy. When compared to the standard drug doxorubicin (g-score: -8.2), the scores of the leads presented slightly lower scores both in the in vitro and in silico studies. But the selected compounds have showed good enough values as these are the leads and in future these can be further refined and can step forward in the drug discovery pipeline.

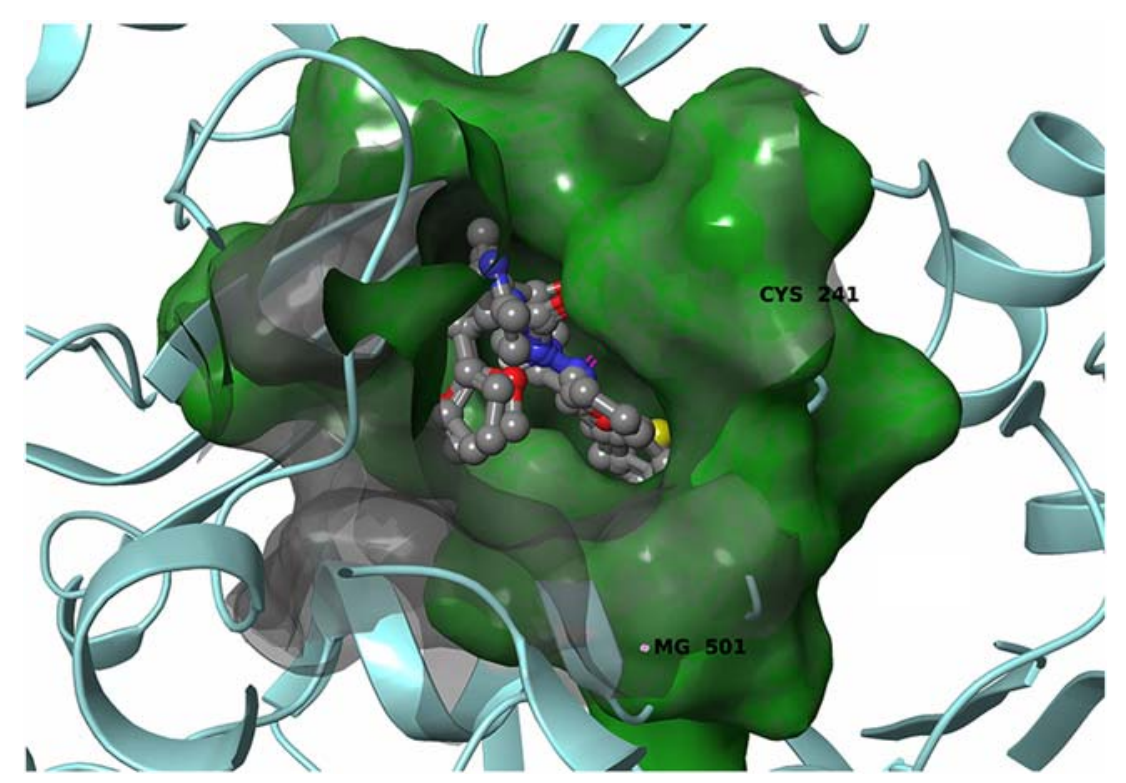

Figure 2. Fitting of the four molecules $(\mathbf{5 a}, \mathbf{5 l}, \mathbf{5 r}$ and $\mathbf{5 s})$ inside the pocket. 
Table 2. Docking, physiochemical and energy scores of the four complexes.

\begin{tabular}{lcccccc}
\hline & & \multicolumn{5}{c}{ ADME } \\
\cline { 3 - 6 } Molecule & G-score & $\begin{array}{c}\text { Molecular } \\
\text { weight }\end{array}$ & $\begin{array}{c}\text { Percent human } \\
\text { oral absorption }\end{array}$ & CNS & $\begin{array}{c}\text { Rule } \\
\text { of five }\end{array}$ & $\begin{array}{c}\text { Prime energy } \\
\text { (K.Cal/mol) }\end{array}$ \\
\hline $\mathbf{5 s}$ & -5.59 & 244.24 & 100 & 0 & 0 & -46.22 \\
$\mathbf{5 r}$ & -5.54 & 260.31 & 100 & 0 & 0 & -49.90 \\
$\mathbf{5 1}$ & -5.52 & 269.30 & 100 & 0 & 0 & -32.88 \\
$\mathbf{5 a}$ & -5.49 & 272.27 & 100 & 0 & 0 & -29.66 \\
\hline
\end{tabular}

\section{Conclusions}

In conclusion, cyclopropane carbohydrazide analogues (5a-t) were prepared from 2-furfuraldehyde via Knoevenagel condensation. Among all the compounds screened for anti-cancer activity, compounds $\mathbf{5 a}, \mathbf{5 l}, \mathbf{5 r}$ and $\mathbf{5} \mathbf{s}$ were considered promising and showed cytotoxicity at micromolar concentration against all the tested cell lines with $\mathrm{IC}_{50}$ values ranging between 1.9-8.45 $\mu \mathrm{M}$ binding modes of these four compounds also produced good results with the anti-cancer target colchicine binding site.

\section{Supplementary Information (SI)}

Supplementary Information is available at www.ias.ac. in/chemsci.

\section{Acknowledgements}

The authors are grateful to Laxai Avanti Life Sciences for providing facilities to carry out the work. The authors are also thankful to the CSIR-Indian Institute of Chemical Technology, Hyderabad for performing the biological activity studies and KLEF University for providing support in conducting the docking studies.

\section{References}

1. Cao W, Zhang H, Chen J, Deng H, Shao M, Lei L, Qian J and Zhu Y 2008 Tetrahedron 646670

2. Toraskar M P, Kadam V J and Kulkarni V M 2010 Int. J. Pharm. Pharma Sci. 2132

3. Baba Y, Saha G, Nakao S, Iwata C, Tanaka T, Ibuka T, Ohishi H and Takemoto Y $2001 \mathrm{~J}$. Org. Chem. 6681

4. Boger D L, Hughes T V and Hedrick M P 2001 J. Org. Chem. 662207

5. Graham D W, Ashton W T, Barash L, Brown J E, Brown R D, Canning L F, Chen A, Springer J P and Rogers E F 1987 J. Med. Chem. 301074
6. Salaun J and Baird M S 1995 Curr. Med. Chem. 2511

7. Yoshida S, Rosen T C, Meyer O G J, Sloan M J, Ye S, Haufe G and Kirk K L 2004 Bioorg. Med. Chem. 12 2645

8. Faust R 2001 Angew. Chem., Int. Ed. 402251

9. Brandt $\mathrm{W}$ and Thiemann T 2003 Chem. Rev. 1031625

10. Yanovskaya L A, Dombrovsky V A and Khusid A Kh 1980 In Tsiklopropanis funktsionalnimi gruppami. Sintez i primenenie. (Cyclopropanes with Functional Groups. Synthesis and Application) (Moscow: Nauka)

11. Tsuji T and Nishida S 1987 In The Chemistry of the Cyclopropyl Group (New York: Wiley \& Sons)

12. Boche G and Walbirsky H M 1990 In Cyclopropane Derived Intermediates (New York: John Wiley)

13. Rappoport Z 1996 In The Chemistry of the Cyclopropyl Group (New York: Wiley \& Sons)

14. Salaün J 2000 In Topics in Current Chemistry; Small Ring Compounds in Organic Synthesis VI: Cyclopropane Derivatives and their Diverse Biological Activities Vol. 207 (Heidelberg: Springer Berlin) pp. 1-67

15. Ellis D, Kuhen K L, Anaclerio B, Wu B, Wolff K, Yin H, Bursulaya B, Caldwell J, Karanewsky D and He Y 2006 Bioorg. Med. Chem. Lett. 164246

16. Yong S R, Ung A T, Pyne S G, Skelton B W and White A H 2007 Tetrahedron $\mathbf{6 3} 1191$

17. Krapcho A P 2007 Arkivoc 21

18. Ziyat H, Ait Itto M Y, Ait Ali M, Riahi A, Karim A and Daran J 2006 Arkivoc 1215

19. Doyle M P and Yan M 2002 Arkivoc 8180

20. Doyle M P and Hu W 2003 Arkivoc 715

21. Barluenga J, Muñiz K, Ballesteros A, Martínez S and Tomás M 2002 Arkivoc 5110

22. Cruz D C, Yuste F, Díaz E, Ortiz B, Sánchez-Obregón S, Walls F and García Ruano J L 2005 Arkivoc 6211

23. Mossman T 1983 J. Immunol. Methods 6555

24. Palla G, Predieri G and Domiano P 1986 Tetrahedron 42 3649

25. Friesner R A, Murphy R B, Repasky M P, Frye L L, Greenwood J R, Halgren T A, Sanschagrin P C and Mainz D T 2006 J. Med. Chem. 496177

26. Schrödinger Release 2015-1: LigPrep, version 3.3, Schrödinger, LLC, New York, NY, 2015

27. Small-Molecule Drug Discovery Suite 2015-1: QikProp, version 4.3, Schrödinger, LLC, New York, NY, 2015

28. Kumar D, Raj K K, Malhotra S V and Rawat D S 2014 Med. Chem. Comm. 5528 